Surprise-induced Deafness

Surprise-induced Deafness: Unexpected Auditory Stimuli Capture Attention to the Detriment of Subsequent Detection

Takashi Obana ${ }^{123}$, Stephen Wee Hun $\mathrm{Lim}^{2}$, \& Christopher L. Asplund ${ }^{12345}$

1 N.1 Institute for Health, National University of Singapore, Singapore

2 Department of Psychology, National University of Singapore, Singapore

3 Division of Social Sciences, Yale-NUS College, Singapore

4 Centre for Sleep and Cognition, Yong Loo Lin School of Medicine, Singapore

5 Neuroscience and Behavioural Disorders, Duke-NUS Medical School, Singapore

Author Note: This work was supported by a Heads and Deanery Research Support Scheme

grant to S.W.H.L, and by a Singapore Ministry of Education and Yale-NUS College start-up

grant to C.L.A. The authors would like to thank Wang Yushi and Lee Sze Chi for their

assistance with data collection, Helen Jin for stimulus preparation, and Garry Kong and Daryl

Fougnie for helpful discussion. Anonymized data from each experiment have been made

publicly available on Figshare and can be accessed at

https://doi.org/10.6084/m9.figshare.19410989.v1. Materials and code are not yet available.

This manuscript is not yet published. Version 2, 30 March 2022. 
Address for correspondence: Christopher L. Asplund, Yale-NUS College, 28 College Avenue West \#01-501, Singapore 138533, chris.asplund@yale-nus.edu.sg

Word count for main text: 16,478

\begin{abstract}
Attention is often captured by unexpected or unusual sounds. Such stimulus-driven control of attention can be adaptive, as potentially relevant events need to be quickly evaluated and acted upon. Attentional capture, however, comes with a cost: Ongoing tasks may be disrupted. In a series of seven experiments $(n=773)$, we investigated the effects of task-irrelevant, rare, and relatively unexpected sounds ("surprise stimuli") on probe detection in rapid auditory presentation (RAP) streams. Surprise stimuli caused "surprise-induced deafness" ( $\mathrm{SiD})$, a substantial detection deficit that lasted for less than a second within each trial and gradually habituated across several trials. SiD was sensitive to informational surprise, with larger deficits following stimuli that were infrequent or varied across trials. The effect also generalized: Natural sounds or constructed stimuli could disrupt probe detection across task difficulties. We compared SiD to the auditory attentional blink (AAB), a similar paradigm in which goal-directed target processing disrupts probe detection. We found that the two deficits were weakly correlated. We conclude that $\mathrm{SiD}$ is a novel perceptual deficit that primarily reflects stimulus-driven attentional capture. It may involve other forms of attentional control as well, thereby reflecting multiple attentional influences on awareness.
\end{abstract}

Key phrases: auditory attention, surprise, stimulus-driven attention, individual differences, attentional capture 


\section{General importance}

When an unexpected sound occurs, it often grabs our attention. Our focus shifts to the sound so that we can assess its importance. The sound could be a warning of imminent danger; alternatively, it could be someone's innocuous ringtone. In this study, we show that there is a cost when a sound grabs our attention. Specifically, we may fail to hear a subsequent sound for which we were listening, even when the unexpected and expected sounds come from the same source. This effect, which we term "surprise-induced deafness", lasts for less than a second after each unexpected sound, and it diminishes as the novelty of such sounds wears off. Our findings could be relevant to the design of user interfaces. More broadly, they improve our theoretical understanding of the trade-off between staying on task and paying attention to what is new. 


\section{Introduction}

An organism's survival depends on its successful detection of unexpected changes in its surroundings. Hearing is especially important for this function because it can serve as an early warning system (Scharf, 1998), sensitive to changes regardless of their location relative to gaze and body position. In addition to these sensory features, the human auditory system includes cognitive processes to rapidly assess sounds for novelty or deviance from expectation (Fritz et al., 2007). A stimulus that warrants further evaluation may automatically become the focus of selective attention, thereby aiding perception and the production of an appropriate behavioral response. In addition to attention's faciliatory roles, however, it has a dark side: Unattended items often fail to reach awareness (Chun \& Marois, 2002). In this study, we tested whether non-spatial attentional capture could produce such deficits in auditory awareness. We also sought to characterize the conditions that influence whether attention is redirected in the first place, thereby allowing us to further elucidate the mechanisms by which attention is controlled.

The auditory system, like other sensory systems, is sensitive to stimulus salience. Many factors can render a stimulus salient, including its novelty, frequency of occurrence, deviance from expectation, physical intensity, or behavioral relevance in a given context (Downar et al., 2002; Fritz et al., 2007). These first three factors involve the idea of informational surprise, as the event carries information by violating ongoing expectations about the world (Asplund, Todd, Snyder, Gilbert, et al., 2010; Asplund et al., 2020; Horstmann, 2015; Meyer et al., 1991; Niepel et al., 1994; Parmentier, 2014). They also involve Bayesian surprise, as the stimulus causes an update to cognition and behavior (Itti \& Baldi, 2009). Brain measures index these changes. For example, novel or deviant sounds evoke an electroencephalographic and magnetoencephalographic response termed the mismatch negativity (MMN) (Escera et al., 1998; Friedman et al., 2001; Näätänen et al., 
2007; Näätänen \& Alho, 1995; Niepel et al., 1994; Parmentier, 2014). The MMN emerges rapidly following stimulus presentation (100-200 ms), after which the evoking stimulus may become attended if further investigation is required (Friedman et al., 2001; Horváth et al., 2008). In the case of a novel stimulus, a subsequently evoked component (the novelty-P3) is thought to index an attentional shift towards the stimulus (Courchesne et al., 1975; Friedman et al., 2001; Friedman \& Simpson, 1994).

Behaviorally, the response to a relatively unexpected or novel stimulus is often orientation towards that stimulus. This "orienting response" (OR) includes positioning the appropriate receptor organ to investigate a potentially relevant stimulus or change in the environment (Pavlov, 1927; Sokolov, 1990). The OR can involve physical reorientation, such as turning one's head to better localize a sound, as well as cognitive reorientation: Attentional resources are reallocated so that the stimulus or event can be better processed (Downar et al., 2002; Escera et al., 1998; Kahneman, 1973). Attentional reallocation may involve a spatial or non-spatial shift of attention, and it may occur in the absence of physical reorientation. The novelty-P3 has been proposed to represent this cognitive aspect of the OR (Escera et al., 2000; Friedman et al., 2001; Knight \& Scabini, 1998). Importantly, both the novelty-P3 and the behavioral components of the OR rapidly habituate as the relative novelty of the evoking stimulus declines, such as following repeated presentations.

Although redirecting attention to a stimulus enhances its processing, there are also costs of attentional shifts; ongoing task performance may suffer. Performance impairments in a primary task are often indicators of auditory distraction or attentional capture (Schröger, 1996). For example, in a task where participants had to quickly report which of two speakers produced a target tone, responses were slowed by the presentation of a novel and taskirrelevant sound (Niepel et al., 1994). Indeed, the presentation of deviant sounds generally slows target detection and discrimination (Dalton \& Lavie, 2004; Horváth \& Winkler, 2010; 
Parmentier, 2014; Roeber et al., 2003; Schröger, 1996; Vachon et al., 2017). In some cases, primary task accuracy may suffer (Horváth \& Winkler, 2010; Schröger, 1996). Such speed and accuracy impairments are contingent on the time between the distracting stimulus and the target. Most impairments follow short (125-200 ms) SOAs (stimulus onset asynchronies) (Horváth \& Winkler, 2010; Niepel et al., 1994; Schröger, 1996; Vachon et al., 2017), with no impairments evidenced following longer SOAs such as $560 \mathrm{~ms}$ (Schröger, 1996) or $1500 \mathrm{~ms}$ (Niepel et al., 1994) SOAs (though see Roeber et al., 2003).

Curiously, to the best of the authors' knowledge, no experiments on the detection costs of auditory attentional capture have examined the habituation of these effects. The costs may not habituate in the paradigms used, or they may show OR-like habituation that is masked due to averaging across trials. Results from many visual attentional capture experiments have analogous uncertainties, though some findings indicate rapid habituation of “surprise" capture (Horstmann, 2005, 2015). In surprise-induced blindness (SiB), for example, a relatively unexpected and task-irrelevant stimulus impairs detection of a subsequent probe presented in a rapid serial visual presentation (RSVP) stream of distractors (Asplund, Todd, Snyder, Gilbert, et al., 2010). The timecourse of SiB is generally similar to the auditory impairments noted above. A potentially important nuance is that although visual attentional capture is observed with short SOAs (100-150 ms), a more severe deficit is evidenced for 350-400 ms SOAs. (No deficit is found for longer SOAs, such as $780 \mathrm{~ms}$ ). Furthermore, only this more severe deficit rapidly habituates across several presentations of surprise stimuli, akin to the habituation profiles for both the orienting response (Kahneman, 1973; Pavlov, 1927; Sokolov, 1990) and the novelty-P3 (Escera et al., 2000; Friedman et al., 2001; Knight \& Scabini, 1998). An auditory version of SiB could be used to test for similar temporal dynamics within and across trials. Such a paradigm is what we propose to develop in the present study. 
An existing paradigm, the auditory attentional blink (AAB), provides both a relevant procedure and additional clues about the temporal aspects of attentional deployment. In the $\mathrm{AAB}$, participants search for a target sound and probe sound in a stream of distractors. The probe is often missed when it closely follows the target. In many AAB studies, performance increases monotonically with increasing SOA (Horváth \& Burgyán, 2011; Mondor, 1998; Potter et al., 1998; Shen \& Mondor, 2006; Vachon \& Tremblay, 2005). In some AAB experiments, however, the most severe deficits occur with SOAs around 300-400 ms (Arnell \& Jolicoeur, 1999; Martens et al., 2009; Tremblay et al., 2005). None of these deficits has been reported to habituate across trials.

Despite the similarities between the auditory attentional blink (AAB) and paradigms that reflect attentional capture by deviant or novel sounds, they critically differ in how attention is allocated to the deficit-inducing stimulus. Traditionally, attention has been thought to be controlled in either a top-down, goal-directed manner or a bottom-up, stimulusdriven one (Debener et al., 2002; Egeth \& Yantis, 1997). The AAB primarily involves the former, as the deficit is absent or greatly attenuated when the first target item is ignored (Arnell \& Jolicoeur, 1999; Shen \& Mondor, 2006). Conversely, attentional capture is often ascribed to the latter form of attentional control (Asplund, Todd, Snyder, Gilbert, et al., 2010; Schröger, 1996).

There has been longstanding debate, however, over whether attentional capture is due exclusively to stimulus-driven factors that are independent of behavioral goals. For example, the contingent capture hypothesis posits that stimuli capture attention only if they contain a feature that defines targets (Folk et al., 1992). The target-defining feature can be general, such as distinctiveness of the target and attention-grabbing stimulus relative to distractors (Bacon \& Egeth, 1994; Egeth \& Yantis, 1997). In such instances, participants can use a "singleton" mode to detect the target. This attentional mode, however, leaves them 
vulnerable to capture by a singleton distractor. Singleton capture and other forms of contingent capture have been demonstrated in audition, though non-contingent capture has been evidenced as well (Dalton \& Lavie, 2004; Vachon et al., 2017).

Another possibility is that the dichotomy of auditory attentional control is incomplete or unhelpful, as has been argued for visuospatial attention (Awh et al., 2012; Bucker \& Theeuwes, 2017; Luck et al., 2020; Pearson \& Le Pelley, 2020). That is, other sources of control, such as selection history, may need to be evoked to explain the range of observed behavioral results. For example, learned stimulus values can cause attentional deployment to be incongruent with either current task goals or the bottom-up properties of the stimulus (Bucker \& Theeuwes, 2017; Pearson \& Le Pelley, 2020). Similarly, the habituation of surprise-induced blindness effects implies that a dynamically-updated internal model influences whether, and to what degree, a stimulus redirects attention. Such capture may nevertheless conflict with the individual's task goals and voluntary control of attention.

\section{The Present Experiments}

In this study, we describe a series of experiments designed to investigate auditory attentional capture by surprising stimuli. In an initial experiment, we attempted to establish and characterize surprise-induced deafness ( $\mathrm{SiD})$, an auditory analogue of surprise-induced blindness in which a relatively rare and unexpected stimulus causes a subsequent probe item to be missed. We also sought to test the temporal properties of any such deficit, both within each trial and across multiple trials containing surprising stimuli. We expected pronounced deficits for SOAs around 300-400 ms, smaller effects for shorter SOAs ( $\sim 100 \mathrm{~ms})$, and no effects for far longer ones (Asplund, Todd, Snyder, Gilbert, et al., 2010; Horváth \& Burgyán, 2011; Meyer et al., 1991; Niepel et al., 1994; Schröger, 1996). We also predicted that SiD

effects, particularly those for intermediate SOAs, would habituate after repeated presentations of surprising items (Asplund, Todd, Snyder, Gilbert, et al., 2010; Friedman et al., 2001; 
Friedman \& Simpson, 1994; Kahneman, 1973; Pavlov, 1927; Sokolov, 2002). Given that auditory novelty-P3s largely attenuate after approximately six stimulus exposures, we expected the habituation rate of the behavioral effects to be similar, which would also make them comparable to effects observed in vision (Friedman \& Simpson, 1994).

In six subsequent experiments, we further explored the SiD phenomenon and its relevance to attentional control. Foremost, it was necessary to simplify the paradigm so that it could be run online using Amazon Mechanical Turk (AMT; Experiment 2). We then tested which aspects of salience modulate or are necessary for $\mathrm{SiD}$. Specifically, we examined the effects of the surprising stimuli's relative novelty and frequency of appearance (Experiment 3), as well as the effects of their gross behavioral relevance and low-level stimulus properties (Experiment 4). We also considered whether participants adopted search strategies that may have introduced more goal-directed attentional elements into the task (i.e., singleton detection mode), as opposed to primarily stimulus-driven components (Experiment 5). Finally, after constructing and testing an auditory attentional blink paradigm (Experiment 6), we conducted a correlation experiment to determine the relationship between surprise-induced deafness and the auditory attentional blink (Experiment 7).

\section{Experiment 1: Establishing and characterizing surprise-induced deafness (SiD)}

In our first experiment, we tested whether the presentation of an unexpected and task-irrelevant auditory stimulus (the surprise stimulus) would impair the detection of a subsequent probe item. We also sought to characterize any such deficit's timecourse, both the expected transience within each trial and the expected habituation across them. Although our primary dependent measure was probe detection rate, we also recorded and analyzed reaction times because auditory masking often fails to eliminate perception (Crowder, 1993; Potter et al., 1998). Finally, we tested for the generalizability of any observed deficits by swapping the stimulus categories of distractors and probes as compared to surprise stimuli (Experiments 1A 
and 1B). These versions of the experiment are presented in sequence, followed by a comparison between them.

To test each of the ideas above, we adapted the surprise-induced blindness ( $\mathrm{SiB}$ ) paradigm (Asplund, Todd, Snyder, Gilbert, et al., 2010) from the visual domain for the auditory domain. We predicted a similar pattern of results for surprise-induced deafness (SiD), namely maximal deficits from intermediate SOAs (300-400 ms), rapid habituation of the effects from such SOAs, and the presence of $\mathrm{SiD}$ regardless of the stimulus categories used.

\section{Experiment 1A: SiD in a tone detection task}

\section{Method}

\section{Participants}

Forty-nine individuals ( 25 females, 20 males, 4 gender not reported, mean age $=$ 22.38 years, age range $=19-27)$ from the National University of Singapore (NUS) community with self-reported normal hearing participated for either payment (S\$5) or participation credit in the NUS Department of Psychology research pool. Attentional capture effects from unexpected stimuli are often large (e.g., $0 \%$ detection following first surprise stimulus versus $81 \%$ baseline detection in surprise-induced blindness (Asplund, Todd, Snyder, Gilbert, et al., 2010)), but we recruited a larger sample in case auditory effects were smaller and to confidently assess effect habituation. With $n=49$ and $80 \%$ power, we could detect a proportion effect size (Cohen's d) of 0.36 (see Transparency and Openness section below). This small-to-medium effect corresponds to $65 \%$ detection following the first surprise compared to an $81 \%$ baseline. The NUS Institutional Review Board approved of the protocol for this experiment and all subsequent experiments reported here.

\section{Stimuli and Apparatus}

The probe stimulus was a $4000 \mathrm{~Hz}$ pure tone, and 21 other pure tones (log-related 
frequencies between 639 and $3175 \mathrm{~Hz}$ ) served as distractors. Unexpected stimuli

("surprises") were spoken letters of the alphabet, excluding W, N, F, S, and X. The letters, spoken by a female native English speaker, were recorded using an Olympus IC recorder and Bose microphone. All sounds were compressed to a duration of $110 \mathrm{~ms}$ (120 ms for practice trials) without changing the pitch in Audacity (The Audacity Team). All sound stimuli were then adjusted so that their maximum amplitudes were equivalent. Based on reports from pilot participants and the experimenters, the tones and spoken letters were perceived to have roughly equivalent volumes when the former were reduced to $15 \%$ of the latter's amplitude; the stimuli were adjusted accordingly. PsychoPy software version 1.81 .03 (Peirce, 2007) and a Dell computer (OPTIPLEX 990) were used to control stimulus presentation and for data logging. Sounds were presented binaurally through TDK headphones (ST 100) with an approximate maximum volume of $70 \mathrm{~dB}$. Participants made their responses through a standard computer keyboard.

\section{Task Procedures}

Each participant-initiated trial began with the presentation of the probe tone to refresh the participant's memory. In pilot studies, we found that including such reminders was important for obtaining reasonable performance from a majority of the participants. A white fixation cross was displayed in the center of the screen until participants pressed any key to commence each trial. A rapid auditory presentation (RAP) of 30 tones then began, with each tone sounding for $110 \mathrm{~ms}$ with a $10 \mathrm{~ms}$ intervening gap (Figure 1A). The onset and offset of each sound included a 2-ms linear amplitude ramp to eliminate clicking or popping of the stream. During $75 \%$ of the trials, the probe was presented after 17 to 27 RAP items. Participants were instructed to press the ' 1 ' key in response to the probe as quickly as possible without sacrificing accuracy. If no response was made during the RAP, participants were prompted by the question "Target?" $300 \mathrm{~ms}$ after the end of the RAP. (Note that we 
referred to the probe as a "target" with our participants. We use the term "probe" in our descriptions here, for both clarity and consistency with Experiments 6 and 7.) Participants pressed the ' 1 ' key if they judged that the probe had been presented and the ' 0 ' key if not.

\section{A Tone task (Letter surprise stimuli)}

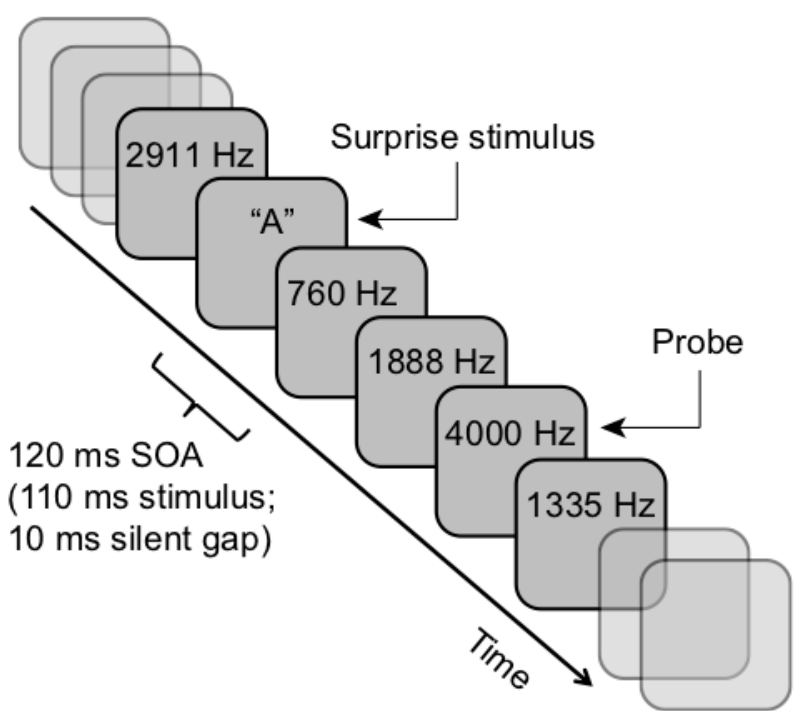

\section{B Letter task (Tone surprise stimuli)}

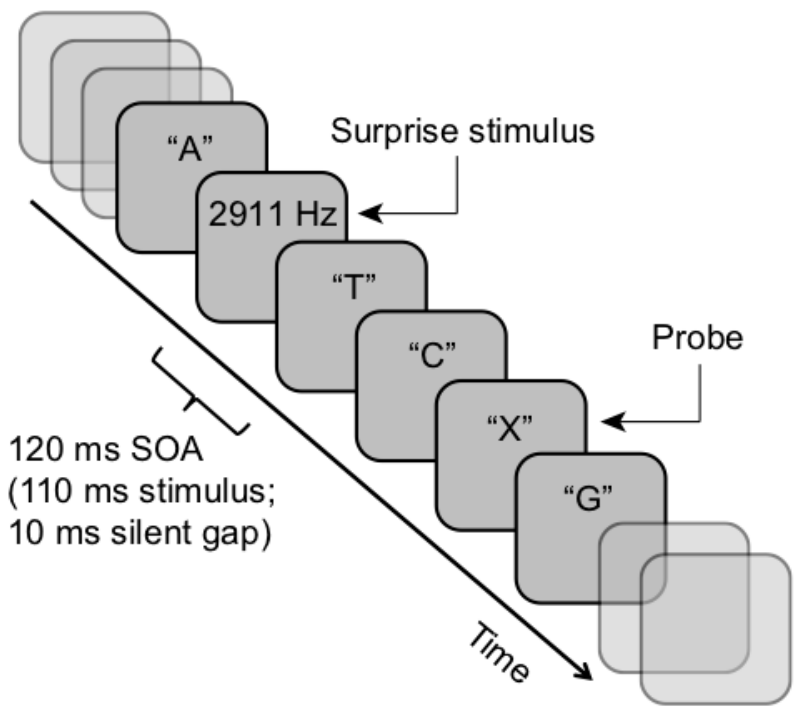

Figure 1 . The sequence of stimuli in a rapid auditory presentation (RAP) stream in (A) Experiment 1A (tone task) and (B) Experiment 1B (letter task). A Lag 3 (360 ms SOA) surprise trial is shown for each. 
During $10 \%$ of the trials, a surprise stimulus replaced a distractor stimulus with a surprise-probe lag of 1,3 , or 8 items $(120,360$, or $960 \mathrm{~ms}$ SOAs $)$ or in the absence of a probe. Each surprise stimulus was randomly chosen from the set of stimuli described above without replacement; a given participant therefore never experienced the same surprise stimulus twice. Each of the four surprise trial types was presented at a random point within each block with three restrictions. First, the initial surprise trial in each block was between trial 6 and 11. Second, there were at least three non-surprise trials between any two surprise trials. This restriction was intended to attenuate any immediate carryover effects from the post-surprise trial (Asplund, Todd, Snyder, \& Marois, 2010) or inter-trial priming effects (Maljkovic \& Nakayama, 1994). Third, the first four surprise trials of each session included one of each type, with an order that was counterbalanced across participants. This consideration ensured that we could examine habituation effects for each surprise-probe lag.

The main experiment consisted of 360 trials divided into 9 blocks of 40, enabling us to characterize a wide range of habituation rates. Between blocks, participants were encouraged to take a short break. Before completing the main experiment, participants completed at least 6 practice trials. No surprises were presented during the practice trials. Most participants completed the session within 60 mins.

\section{Transparency and Openness}

For this and subsequent experiments, we provide explanations for sample size determination, data exclusions, experimental manipulations, and dependent measures. The study's analysis and design were not preregistered; we instead rely on internal and external replications to determine effect reliability and size. Data preparation, visualizations, and statistical analyses for all experiments were implemented in RStudio version 1.0.153 (R Foundation for Statistical Computing) running R version 3.2.4. Power analyses were 
conducted using power.prop.test() and pwr.t.test() in the pwr package, version 1.3-0.

Anonymized data from each experiment have been made publicly available on Figshare and can be accessed at https://doi.org/10.6084/m9.figshare.19410989.v1. Materials and code are not yet available.

\section{Statistical Approach}

Prior to conducting formal analyses on each experiment, we identified individuals who had poor baseline performance or who had numerous trials with multiple responses. Inferring surprise-related deficits from such individuals' performance would be difficult or impossible, so their data were removed from each sample. Each participant's detection performance on non-surprise trials was represented as a d-prime score, with extreme hit or false alarm rates accommodated by setting these values to 0.1 or 0.9 for the $\mathrm{z}$-score transformation (Stanislaw \& Todorov, 1999). In Experiment 1, d-prime values less than approximately 0.5 corresponded to performance indistinguishable from chance responding; we used this threshold for subsequent experiments as well. Inspection of the relevant histograms revealed that only a few individuals had numerous trials with multiple responses, whereas most individuals had $0-3$. We therefore used 10 trials with multiple responses as a cutoff threshold. These data removals applied to both detection and reaction time analyses. For the latter, we also removed individual trials with reaction times over 5 seconds to minimize the effect of outliers.

For Experiment 1 and most subsequent experiments, we constructed and fit generalized linear mixed-effects models (GLMMs). These models allowed us to examine trends in performance across time. Correct detection (hit) rates were assessed using logistic regression (binomial error distribution) implemented with glmer() in the lme4 package (version 1.1.13) (Bates et al., 2015). False alarms were assessed in the same way in a separate model. Reaction times were assessed using glmer() with a gamma error distribution. For the 
models that assessed hits and reaction times, the independent variables were trial type (4 levels: Lag 1, 3, 8, or probe-only; categorical) and time (block number; continuous, 0 centered in the model). For subsequent experiments, the time variable was set up by first labelling each trial with its serial position within the session; this assignment was done separately by trial type, after which the values were normalized to the range -0.5 to 0.5 . The models for false alarms contained the same independent variables (trial type and time), but the two trial type levels were "surprise only" and "no probe, no surprise". Since all factors in each of these models were manipulated within-subjects, the random effects structure included by-subject random intercepts and slopes for the trial type variable. Including by-subject random slopes for time or the Trial Type $\mathrm{x}$ Time interaction produced models that frequently failed to converge, so the random effects structure used is the maximum supported by the design and data (Barr et al., 2013). For models comparing results across experiments, we included each of the factors above, added a between-groups factor of experiment, and used the same random effects structure as before. For all GLMMs, main effects and interactions were assessed using Type II Wald chi-square tests via the Anova() function in the car package (version 2.1.5) (Fox \& Weisberg, 2011).

Post-hoc tests were conducted using the emmeans(), emtrends(), and contrast() functions in the emmeans package (version 1.3.4) (Lenth et al., 2019). Pairwise differences between estimated marginal means and trends were assessed using t-distributions with the degrees of freedom based on Satterthwaite's method. To account for multiple comparisons, we applied Bonferroni correction to each set of post-hoc comparisons; each p-value was multiplied by the number of comparisons in the set.

\section{Results}


Twelve participants ( $24 \%$ of original sample) had either poor baseline detection performance (11), excessive trials with multiple responses (4), or both. After their removal, 37 participants remained in the final sample.
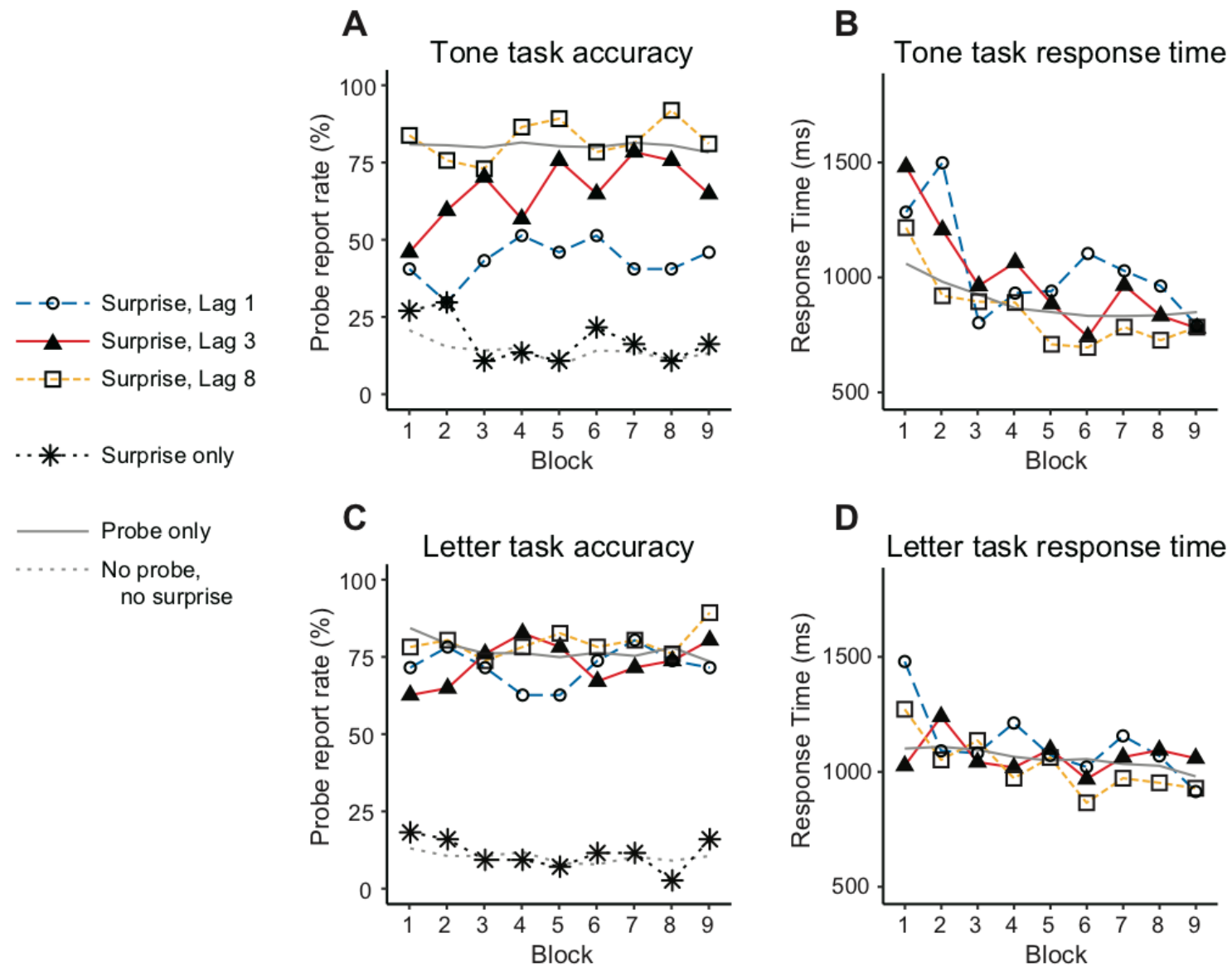

Figure 2. Average probe performance for each trial type by block. (A) Probe report rate in Experiment 1A (tone task). Note that these values are false alarm rates in the "surprise only" and "no probe, no surprise" categories. (B) Probe response time in Experiment 1A. (C) Probe report rate in Experiment 1B (letter task). (D) Probe response time in Experiment 1B. Error bars have been omitted for clarity.

\section{Probe Report}

For visualization purposes, the average probe report rate was calculated for each trial 
type and block (Figure 2A). Generalized linear mixed-effect models (GLMMs, see above) with within-subject variables of time (Block 1 to 9) and trial type (probe-only, Lag 1, Lag 3, Lag 8) were used for analysis. For hits, there was a significant main effect of trial type and a significant Time $\mathrm{x}$ Trial Type interaction (Table 1). The hit rate for Lag 1 was significantly lower than for other trial types ( $p$ s $<.001$ ). The Lag 3 rate was lower than the Lag 8 rate, though not significantly $(p=.059)$. The other two pairwise differences were not significant $(p s>.10)$. The Lag 3 deficit habituated more quickly than probe-only $(p=.014)$, which was due to a significant positive slope for Lag 3 (17\% increase in odds of correct response per block; $p=.015)$ and no significant change over time for probe-only $(p=1)$. No other slopes or slope differences were significant $(p \mathrm{~s}>.85)$. To better understand the habituation at Lag 3 , we compared Lag 3 and probe-only hit rates for each block with Wilcoxon signed ranks tests owing to the binary dependent measure. After Bonferroni correction, only the deficit in Block $1($ mean $=34.9 \%, \mathrm{SD}=50.7 \%)$ remained significant $(p=.013 ; p \mathrm{~s}>.25$ for all others $)$.

For false alarms, the model's random effects structure was simplified to random intercepts only to enable convergence. There was a significant main effect of time $\left(\chi^{2}(1)=\right.$ $12.98, p<.001$ ), with the false alarm rate decreasing across blocks. Neither the main effect of trial type nor the Time $\mathrm{x}$ Trial Type interaction was significant $(p s>.077)$. Surprise-only trials, particularly in early blocks, contained numerically more false alarms, so it is unlikely that either overall surprise effects or the habituation of the Lag 3 effects were due to response bias. By the same token, the low false alarm rate indicated that there were relatively few responses to the surprise stimulus itself.

Table 1. Statistical results for GLMMs assessing probe hit rates in Experiments $1 \mathrm{~A}$ and $1 \mathrm{~B}$ as a function of time and trial type. For Experiment 1B and cross-experiment comparisons, the models' random effects structure was simplified to random intercepts only to enable 
convergence. The GLMM comparing the two experiments included experiment as a factor as well. Significant effects are indicated in bold. $\mathrm{dfs}=$ degrees of freedom.

\begin{tabular}{|c|c|c|c|c|c|c|c|}
\hline & \multicolumn{3}{|c|}{ Expt. 1A (tone } & \multicolumn{2}{|c|}{ Expt. 1B (letter } & \multicolumn{2}{|c|}{ Both Expts. } \\
\hline & \multicolumn{3}{|c|}{ task) } & \multicolumn{2}{|c|}{ task) } & & \\
\hline & dfs & $\chi^{2}(\mathrm{dfs})$ & $p$ & $\chi^{2}(\mathrm{dfs})$ & $p$ & $\chi^{2}(\mathrm{dfs})$ & $p$ \\
\hline Time & 1 & $<.001$ & .99 & 21.11 & $<.001$ & 12.15 & $<.001$ \\
\hline Trial Type & 3 & 55.65 & $<.001$ & 13.00 & .005 & 196.58 & $<.001$ \\
\hline Experiment & 1 & & & & & 0.96 & .33 \\
\hline Time $\times$ Trial Type & 3 & 11.33 & .010 & 11.57 & .009 & 20.99 & $<.001$ \\
\hline Trial Type $\times$ Experiment & 3 & & & & & 101.20 & $<.001$ \\
\hline Time $\times$ Experiment & 1 & & & & & 8.40 & .004 \\
\hline Time $\times$ Trial Type $\times$ & 3 & & & & & 0.31 & .96 \\
\hline Experiment & & & & & & & \\
\hline
\end{tabular}

In surprise-induced blindness, the Lag 3 deficit's habituation was often complete within 2-4 trials (Asplund, Todd, Snyder, Gilbert, et al., 2010; Asplund, Todd, Snyder, \& Marois, 2010). To test for such rapid effects in the present experiment, we examined performance during the Lag 3 trials in the first block. Each participant experienced one Lag 3 trial in the first four surprise trials. Therefore, we calculated the detection rate as a function of the ordinal number of the Lag 3 trial relative to other surprise trials $\left(1^{\text {st. }}: 29 \%, 2^{\text {nd. }}: 56 \%, 3^{\text {rd. }}\right.$ : 
$\left.27 \%, 4^{\text {th. }}: 60 \%\right)$. No clear trend was evident in the detection rates, and a non-parametric

Cochran Q test found no significant differences across the four categories $(\mathrm{Q}(3)=3.46, p$

$=.33$ ). Surprise-induced deafness' habituation appears to be gradual, such that variance overwhelms within-block habituation trends.

\section{Reaction Times}

Only trials with correct probe detection were used for reaction time (RT) analyses. For visualization purposes, mean RTs for hits were calculated for each trial type and block (Figure 2B). Formal analysis revealed significant main effects of time and trial type as well as a significant Time $\mathrm{x}$ Trial Type interaction (Table 2). RTs in the Lag 1 and Lag 3 conditions were significantly longer than in the other two conditions $(p s<.019)$, whereas other pairwise comparisons were not significant $(p \mathrm{~s}=1.00)$. Although RTs significantly decreased for the Lag 3, Lag 8, and probe-only conditions across time ( $p$ s $<.001$; Lag 1: $p=.12)$, only the Lag 3 and probe-only trials had significantly different slopes ( $p=.020 ; p \mathrm{~s}>.49$ for all others).

Table 2. Statistical results for GLMMs assessing reaction times for probe hits in Experiments $1 \mathrm{~A}$ and $1 \mathrm{~B}$ as a function of time and trial type. To enable convergence for Experiment 1B's model, the random effects structure was simplified to random intercepts only, and a loglinked Gamma error distribution was used. Significant effects are indicated in bold. dfs $=$ degrees of freedom.

Exp. 1A (tone task)

Exp. 1B (letter task)

\begin{tabular}{lccccc}
\hline & $\mathrm{dfs}$ & $\chi^{2}(\mathrm{dfs})$ & $p$ & $\chi^{2}(\mathrm{dfs})$ & $p$ \\
\hline Time & 1 & $\mathbf{2 0 2 . 4 0}$ & $<.001$ & $\mathbf{8 5 . 9 4}$ & $<.001$ \\
Trial Type & 3 & $\mathbf{2 6 . 6 9}$ & $\mathbf{< . 0 0 1}$ & $\mathbf{9 . 8 5}$ & $\mathbf{. 0 2}$
\end{tabular}


Experiment 1

$\begin{array}{lllllll}\text { Time } \times \text { Trial Type } & 3 & \mathbf{1 1 . 4 3} & \mathbf{. 0 1} & \mathbf{1 4 . 6 9} & \mathbf{. 0 0 2}\end{array}$

\section{Experiment 1B: Surprise-induced deafness in a letter detection task}

\section{Method}

Experiment 1B was conducted concurrently with Experiment 1A, and the experiments were identical with the following exceptions. Fifty-three participants (29 females, 21 males, 3 gender not reported; mean age $=21.78$ years, age range $=19-30$ ) searched for a spoken letter "X" probe amongst spoken letter distractors. Surprise stimuli were pure tones, and each participant experienced a given tone frequency only once.

\section{Results}

Eight participants ( $15 \%$ of the sample) had either poor baseline detection performance (4), excessive trials with multiple responses (5), or both. After their removal, 45 participants remained in the final sample.

\section{Probe Report}

The pattern of results was similar to Experiment 1A's, though the effects of each variable were generally weaker (Figure $2 \mathrm{C}$ ). The main effect of time, the main effect of trial type, and the Trial Type $\mathrm{x}$ Time interaction were all significant (Table 1). Unlike in the previous experiment, however, the only significant pairwise comparisons reflected lower Lag 1 performance relative to either Lag $8(p=.033)$ or probe-only $(p=.048)$ trials $(p s>.11$ for other comparisons). Lag 3 and probe-only slopes across time did not significantly differ ( $p$ $=.064$ ), and the suggestion of an effect was driven by a numerically small but significant decrease in the probe-only hit rate $(p<.001)$. No other slopes or slope differences were significant $(p \mathrm{~s}>.29)$. 
For false alarms, there was a significant main effect of time $\left(\chi^{2}(1)=4.22, p=.040\right)$, with the rate decreasing across blocks. There was no significant main effect of trial type and no significant Time $\mathrm{x}$ Trial Type interaction $(p \mathrm{~s}>.51)$.

As we had done for Experiment 1A, we analyzed the probe detection rate for the first block's Lag 3 trials as a function of surprise stimulus experience. There was no apparent trend to performance $\left(1^{\text {st: }}: 64 \%, 2^{\text {nd. }}: 73 \%, 3^{\text {rd. }}: 75 \%, 4^{\text {th. }}: 36 \%\right)$, and a Cochran $Q$ test found no significant difference across trials $(\mathrm{Q}(3)=4.49, p=.21)$.

\section{Reaction Times}

The pattern of results was broadly similar to that in Experiment 1A, albeit with weaker surprise effects (Figure 2D). The main effect of time, the main effect of trial type, and the Time $\mathrm{x}$ Trial Type interaction were all significant (Table 2). Participants became faster overall as the experiment progressed, with significant RT decreases for all trial types ( $p$ s $<.001)$ save Lag $3(p=1.00)$. Lag 1 RTs decreased more quickly than either Lag $3(p=.035)$ or probe-only RTs $(p=.039)$. No other pairwise comparisons of slopes were significant ( $p$ s $>$.064). RTs did not significantly differ between pairs of trial types $(p \mathrm{~s}>.091)$ save Lag 1 compared to probe-only $(p=.028)$.

\section{Probe Report Across Experiments $1 A$ and $1 B$}

Performance across the experiments was assessed by adding an independent variable for experiment to our GLMMs (Table 1). Both the Experiment $\mathrm{x}$ Trial Type and Experiment $\mathrm{x}$ Time interactions were significant, whereas the three-way Time $\mathrm{x}$ Trial Type $\mathrm{x}$ Experiment interaction was not. Performance for Lag 1 was lower in Expt. 1A than in 1B $(p<.001$; Figures $2 \mathrm{~A}, 2 \mathrm{C}$ ), but no other trial type differences across experiments were significant ( $p \mathrm{~s}$ $>$.13). Probe-only trials differed in their slopes across experiments $(p=.006)$, driven by the aforementioned decrease in performance across time in Experiment 1B. No other trial type slopes significantly differed across experiments $(p s>.36)$. There were more false alarms in 
the tone task (Experiment 1A), though this difference did not produce a significant main effect of experiment $\left(\chi^{2}(1)=2.85, p=.092\right)$. For false alarms, no interactions with experiment were significant $(p s>.30)$. For cross-experiment RT comparisons, all tested models with random effects failed to converge.

\section{Discussion}

In this first experiment, we demonstrated that relatively rare and unexpected auditory stimuli can induce a detection deficit. This deficit, which we term surprise-induced deafness (SiD), has many characteristics that are similar to its visual counterpart, surprise-induced blindness (Asplund, Todd, Snyder, Gilbert, et al., 2010). In both $\mathrm{SiD}$ and $\mathrm{SiB}$, the impairment in probe detection depends on the time between the surprise stimulus and the probe, with larger deficits at Lags 1 and 3. The effects in these two conditions can be distinguished by the habituation rates, with more rapid reduction of the Lag 3 effects in each paradigm.

Nevertheless, we also found substantial differences across the two modalities. SiD was a less severe deficit initially, but the rate of habituation was also considerably slower than SiB's. Furthermore, SiD only partially generalized to different classes of stimuli. Below, we consider each of these features for the Lag 1 and Lag 3 deficits, as well as how they will be explored in subsequent experiments in this study.

For the tone task (Experiment 1A), there was a persistent and severe deficit at Lag 1. One possibility is that this deficit reflects perceptual processes, such as auditory masking (Jesteadt et al., 1982; Moore \& Glasberg, 1981). Auditory masking persists across hundreds or even thousands of trials (Delahaye et al., 1999). Furthermore, a dynamic letter may be a more effective mask for a tone than vice versa, which would be consistent with the smaller Lag 1 deficit in Experiment 1B. Alternatively, the Lag 1 deficit in Experiment 1A may represent an attentional effect that habituates slowly, akin to what was observed in surpriseinduced blindness (Asplund, Todd, Snyder, Gilbert, et al., 2010). 
The Lag 3 deficit and its habituation were also clear only in Experiment 1A. The observed habituation is consistent with attentional reallocation due to an orienting response (Kahneman, 1973; Pavlov, 1927; Sokolov, 2002). As Lag 3 corresponds to an SOA of 400 ms, the deficit's timecourse within each trial is also consistent with delayed attentional capture following a surprising event (Asplund, Todd, Snyder, Gilbert, et al., 2010; Horstmann, 2005, 2015; Theeuwes et al., 2000). In addition, it is consistent with the maximal attentional blink effects that occur between 200-500 ms after the inducing target item. This timing is characteristic of the visual AB (Dux \& Marois, 2009), and it is frequently found for the auditory AB as well (Arnell \& Jolicoeur, 1999; Martens et al., 2009; Tremblay et al., 2005). Taken together, the features of the Lag 3 deficit suggest that it is sensitive to informational surprise, unlike the Lag 1 effects. Surprise-induced deafness (SiD) therefore refers to the former effect, and we investigate Lag 3 effects in subsequent experiments.

Despite the robust habituation of $\mathrm{SiD}$, the rate of habituation was slower than we had anticipated. Based on surprise-induced blindness (Asplund, Todd, Snyder, Gilbert, et al., 2010) and auditory novelty-P3 habituation rates (Friedman et al., 2001), we had expected complete attenuation after 2-6 presentations of the surprising stimulus. Attenuation in Experiment 1A, however, was not convincingly complete until at least 10-15 stimuli had been experienced. One possibility is that the surprise stimuli were perceived as heterogenous, and such varying stimuli yield slower and incomplete habituation (Asplund, Todd, Snyder, Gilbert, et al., 2010; Asplund, Todd, Snyder, \& Marois, 2010). We explore the effects of same and varying surprise stimuli in Experiments 2 and 3. These experiments also allow us to explore the possibility that participants anticipate and inhibit the surprise stimuli so that they have reduced effects over repeated presentations.

Finally, the differences between Experiments 1A and 1B suggest that stimulus features contribute to SiD. One possibility is that letters are more salient because they are 
more meaningful than pure tones. We investigate the role of the surprise stimuli's content in Experiment 4. Alternatively, spoken letters may have different effects in RAP streams because they are dynamic — a series of tones is arguably more like a series of visual stimuli, each of which is static — or because our stimulus equalization method did not work fully as intended. We use a different method in Experiment 5.

\section{Experiment 2: A simplified surprise-induced deafness paradigm}

Having demonstrated the surprise-induced deafness effect, we next developed a simplified and abbreviated version of the paradigm. We focused on the Lag 3 effects in the tone task, which evidenced both a strong initial deficit and habituation across surprise trials. We used this new paradigm for the two aims of Experiment 2. First, we sought to replicate the salient features of $\mathrm{SiD}$ in a paradigm that could be adapted for use on Amazon Mechanical Turk (AMT), an online platform that can be used to conduct cognitive psychology experiments (Crump et al., 2013). Given that SiD is calculated from rather few trials, a large sample size would be needed for subsequent investigations into higher-order effects. Second, we informally tested whether SiD would more quickly habituate when the same surprise stimulus was used for each surprise trial. Successive presentations of the surprise stimulus would contain less novelty, potentially reducing its ability to capture attention. Surprise-induced blindness has been found to habituate more rapidly under such circumstances (Asplund, Todd, Snyder, Gilbert, et al., 2010; Asplund, Todd, Snyder, \& Marois, 2010). In this experiment, we tested whether significant deficit habituation, perhaps even its complete elimination, could be observed in a simplified and abbreviated paradigm. In Experiment 3, we explored habituation rates formally using AMT.

\section{Method}

The procedure for Experiment 2 was broadly similar to Experiment 1's, with numerous specific changes to address the new experiment's goals and to improve the SiD 
paradigm. Twenty-two participants ( 7 males, 15 females, mean age $=20.55$, age range $=18$ 24) completed a 20-minute session of 120 trials in three blocks. The Lag 1 and 8 trials from Experiment 1A became Lag 3 trials in the present experiment, thereby producing the same total number of Lag 3 surprise trials as in Experiment 1 (9). Given the medium-to-large effect size for the first block's Lag 3 deficit in Experiment 1A (Cohen's d=0.69), a sample size of 20 would yield $80 \%$ power for the surprise effect. We also anticipated significant, if not complete, habituation within the session.

Each participant was presented with the same surprise stimulus in each surprise trial. The surprise stimuli were spoken letters, with each assigned randomly to a participant (e.g., 'A' for the first participant, 'B' for the second participant, etc.). Instead of equilibrating stimulus volumes by matching maximum amplitudes, all stimuli were equilibrated by dividing each waveform by its summed absolute amplitude (envelope). This procedure resulted in sound intensities that we judged to be subjectively similar. We employed this approach for subsequent experiments as well. Before each trial, participants could play the probe as many times as they wished. Due to the multiple responses in some trials in Experiment 1, participants were explicitly warned not to respond more than once per trial if multiple responses were detected.

\section{Results and Discussion}

One participant ( $5 \%$ of the sample) was removed from further analysis owing to poor baseline detection performance, leaving 21 participants in the final sample. Warning messages were effective at reducing the incidences of multiple responses; no participant had more than four trials with multiple responses.

For visualization purposes, the average probe report rate was calculated for each trial type and block (Figure 3A). The Time $\mathrm{x}$ Trial Type interaction for hits was significant $\left(\chi^{2}(1)=\right.$ $10.63, p=.001)$, though neither main effect was $(p s>.73)$. Performance increased across 
time for Lag 3 trials $(p=.004)$ but not for probe-only trials $(p=.67)$. As assessed with Wilcoxon signed ranks tests, the hit rate for Lag 3 was significantly lower than the hit rate for probe-only in Block $1(p<.049)$ but hit rates did not differ in subsequent blocks $(p s>.34)$. Within the first block, the hit rate by surprise trial $\left(1^{\text {st. }}: 55 \%, 2^{\text {nd. }}: 59 \%, 3^{\text {rd }}: 69 \%, 4^{\text {th }}: 68 \%\right)$ did not significantly differ (Cochran $\mathrm{Q}(3)=0.93, p=.82)$, consistent with the gradual habituation of a modest initial deficit. For false alarms, there was a significant main effect of time $\left(\chi^{2}(1)\right.$ $=6.89, p<.009)$, as the false alarm rates decreased across blocks. Neither the main effect of trial type nor the Time $\mathrm{x}$ Trial Type interaction was significant $(p s>.20)$. As such, the habituation of the Lag 3 surprise effects was not due to response bias or participants mistakenly reporting the surprise stimulus as the probe.

For reaction times, there was a significant main effect of time $\left(\chi^{2}(1)=4.94, p\right.$ $=.026)$, as responses became faster over the course of the experiment. Neither the main effect of trial type nor the Time $\mathrm{x}$ Trial Type interaction was significant $(p s>.20)$. In subsequent experiments, we focused on detection accuracy because this metric was more robust and aligned more clearly with our interests in awareness. Indeed, conscious detection is the critical measure for related effects such as surprise-induced blindness (Asplund, Todd, Snyder, Gilbert, et al., 2010) and the auditory attentional blink (Horváth \& Burgyán, 2011; Mondor, 1998; Shen \& Mondor, 2006; Vachon \& Tremblay, 2005, 2006). 

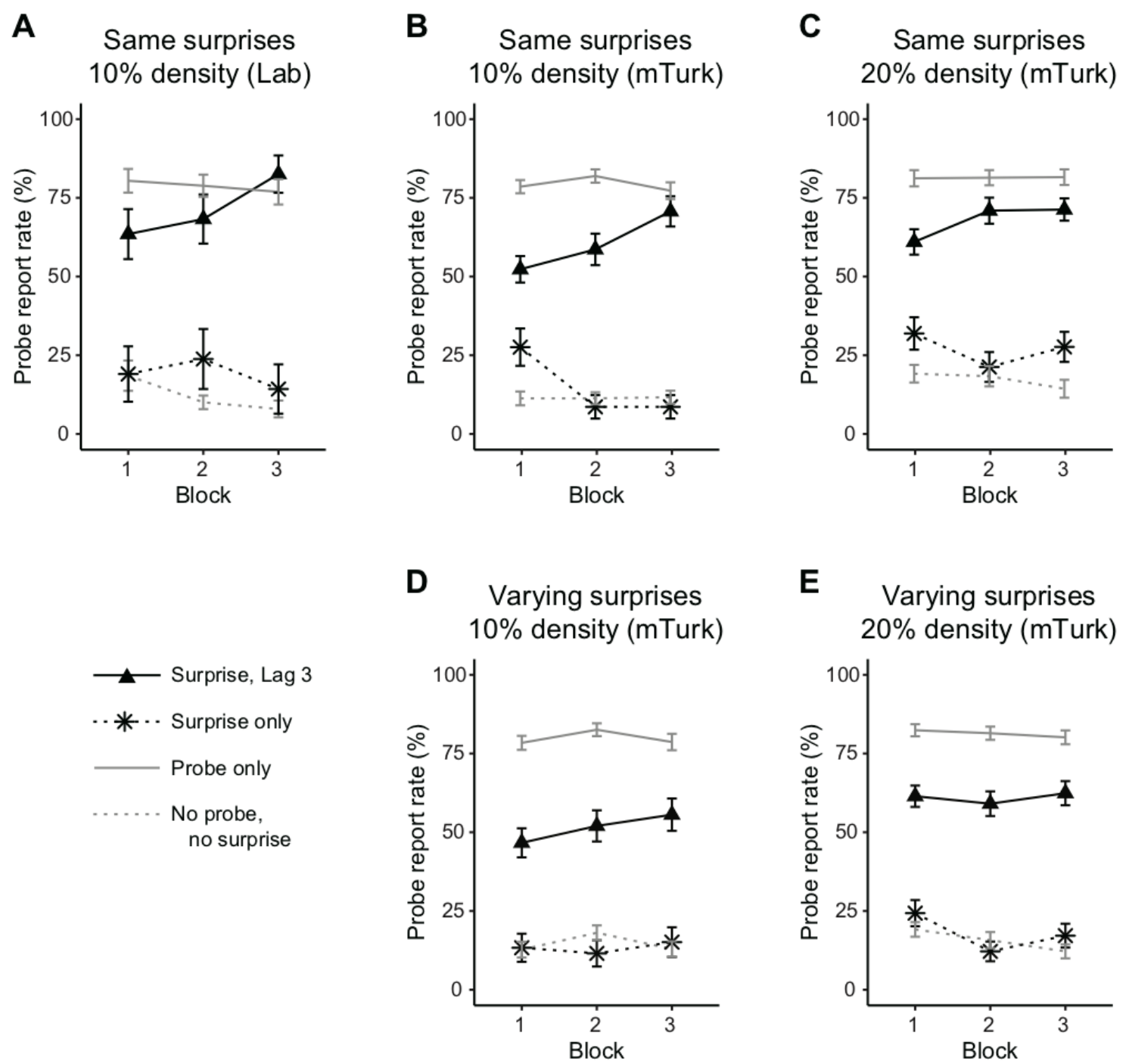

Figure 3. Mean probe report rates for each Trial Type plotted by block in Experiment 2

(Panel A) and Experiment 3 (Panels B-E). Note that these values are false alarm rates in the "surprise only" and "no probe, no surprise" categories. Panels A and B show the effect of the testing platform (lab-based or online), whereas other comparisons illustrate the effects of surprise stimulus heterogeniety (Panels B and C versus D and E) and density (Panels B and D versus $\mathrm{C}$ and $\mathrm{E}$ ). For all panels, error bars represent \pm 1 standard error of the mean (SEM).

\section{Experiment 3: Surprise stimulus novelty and rarity}


In Experiment 2, we used a simplified paradigm to replicate the key features of $\mathrm{SiD}$, both the overall deficit and its habituation. In Experiment 3, we leveraged this simplified design and Amazon Mechanical Turk (AMT) to better understand the effects of novelty and presentation frequency on SiD magnitude. Novelty and rarity are both features that render stimuli more salient (Downar et al., 2002; Horstmann, 2015). Accordingly, novel or relatively rare auditory distractors often grab attention, thereby disrupting task performance (Asplund et al., 2020; Meyer et al., 1991; Niepel et al., 1994; Parmentier et al., 2011; Vachon et al., 2012).

As participants perform the SiD task, they form expectations about the events that are likely to occur within it. Novel or rare stimuli generally deviate from these expectations; they are therefore surprising in an information-theoretic sense (Asplund et al., 2020; Donchin, 1981; Niepel et al., 1994; Parmentier, 2014). Surprises are often psychologically important as well, causing changes to ongoing behavior or updates to expectations (Donchin, 1981; Itti \& Baldi, 2009; Meyer et al., 1991; Niepel et al., 1994). In a SiD task, each surprise stimulus may disrupt probe detection, and it may lead participants to update their expectations about the type and frequency of distractions. The former effect results in SiD, whereas the latter accounts for SiD's habituation.

In the present experiment, we manipulated the relative novelty and rarity of surprise stimuli across groups. We expected $\mathrm{SiD}$ to begin at the same magnitude for each group, diverging as participant expectations changed. Specifically, we hypothesized that individuals presented with the same surprise stimuli across trials would develop more specific expectations and therefore experience less SiD. We also predicted faster effect habituation for these individuals, whereas habituation would remain incomplete for those presented with changing surprise stimuli, as observed in vision (Asplund, Todd, Snyder, Gilbert, et al., 2010; Asplund, Todd, Snyder, \& Marois, 2010). Similarly, we hypothesized that individuals presented with surprise stimuli more frequently would come to expect such events and 
experience less $\mathrm{SiD}$. They would update their expectations more quickly across the course of the experiment - perhaps even from surprise trial to surprise trial—leading to faster effect habituation as well. In addition to testing the hypotheses above, we compared the results from Experiments 2 and 3 to understand the similarities and differences across experimental platforms.

\section{Method}

\section{Participants}

Three hundred and five participants (see Table 3) with normal hearing participated for payment (\$1) via Amazon Mechanical Turk (AMT), an online crowdsourcing platform. On AMT, experimenters ("requesters") post human intelligence tasks (HITs) that anonymous potential participants can choose to complete. To ensure the quality of data, participants were required to have $90 \%$ of their previous HITs approved by the requester and to have at least 1,000 HITs completed (Peer et al., 2014; Summerville \& Chartier, 2013).

The use of AMT made quickly collecting a large sample size feasible. As we had few expectations about the effect sizes for our between-group comparisons for heterogeneity and density, we planned to collect data until each group had at least 40 participants (double the total number of participants in Experiment 2); recruitment was faster than anticipated, so we collected a rather larger sample. Each participant was randomly allocated to one of four groups (Table 3). Participants within each group experienced different surprise stimulus properties, two levels each for the variables heterogeneity and density. For heterogeneity, participants could experience either the same surprise stimulus for each surprise trial (same) or a different stimulus for each trial (varying). For density, surprise trials were either $10 \%$ (4 of 40 ) or $20 \%$ ( 8 of 40 ) of the total number of trials within each of three blocks.

Table 3. Participant information for Experiment 3. The data from participants with low probe 
detection performance $\left(\mathrm{d}^{\prime}<0.5\right)$ were removed from the sample. Group sizes are unequal because each participant was assigned a group randomly. $\mathrm{NR}=$ not reported, $\mathrm{m}=$ males, $\mathrm{f}=$ females.

\begin{tabular}{cccccc}
\hline Heterogeneity & Density & Recruited & Ages & Removed & Final \\
& & sample size & sample \\
& & & & size \\
\hline Same & $10 \%$ & $70(36 \mathrm{~m}, 34 \mathrm{f})$ & $30.93(21-62), \mathrm{NR}=1$ & $12(17 \%)$ & 58 \\
Same & $20 \%$ & $64(36 \mathrm{~m}, 28 \mathrm{f})$ & $30.31(21-60), \mathrm{NR}=2$ & $17(27 \%)$ & 47 \\
Varying & $10 \%$ & $79(43 \mathrm{~m}, 36 \mathrm{f})$ & $31.78(20-52), \mathrm{NR}=1$ & $23(29 \%)$ & 56 \\
Varying & $20 \%$ & $92(58 \mathrm{~m}, 34 \mathrm{f})$ & $30.85(18-65), \mathrm{NR}=1$ & $22(24 \%)$ & 70 \\
\hline Total & & $305(173 \mathrm{~m}, 132$ & $30.97(18-65), \mathrm{NR}=5$ & $74(24 \%)$ & 231 \\
& & & & & \\
\hline
\end{tabular}

\section{Stimuli and Apparatus}

The experiment itself was conducted on Amazon Mechanical Turk (AMT). Previous studies have shown that AMT-based experiments can replicate the visual attentional blink and similar cognitive tasks (Crump et al., 2013). Once individuals on AMT had agreed to participate in our experiment, they were redirected to an online survey system (Qualtrics; Provo, Utah, USA) through which stimuli were presented and the task flow was controlled. At the end of the experiment, participants received instructions to enter a unique code generated by Qualtrics in the AMT HIT to verify that they completed the study to receive 
payment.

The presented stimuli were identical to those used in Experiment 2, except that we expanded the set of surprise stimuli. In addition to the sounds used before, the surprise stimulus set included the following sounds: alarm, balloon popping, car horn, cat meowing, cough, cowbell, dog barking, giggle, hiccup, hi-hat cymbal, lightbulb breaking, mosquito buzzing, plunger, slide whistle, slurping, snare drum, sneeze, tongue popping, “one”, and "two". The spoken digits were recorded from a female native English speaker, whereas the other sounds were drawn from freesound.org (Font et al., 2013). All sounds were compressed to $110 \mathrm{~ms}$ each, with care taken to preserve intelligibility.

\section{Task Procedures}

The task procedures were identical to Experiment 2 with the following exceptions. Foremost, each participant was allocated to one of four groups with different surprise trial features, as described above. The probe response was unspeeded and made at the conclusion of the stream. Before beginning the main experimental blocks, participants completed six practice trials of the task at a slightly slower speed (120 ms SOAs) and another 6 practice trials at normal speed (110 ms SOAs). These practice trials contained feedback. During the practice period, participants were also instructed to adjust the volume to a comfortable level, and headphones were recommended.

Owing to randomization limitations with Qualtrics, a limited number of trial orders were used. All participants in the Same 10\% and Varying 10\% groups experienced the same sequence of trials (only the surprise stimuli were different), whereas the Varying $20 \%$ and Same $20 \%$ groups used two different orders, allowing us to assess whether particular stimulus orders confounded our results. All trial sequences were visually inspected to ensure a reasonable distribution of trial types across time. Critically, the number of trials for each trial type was consistent across blocks. They also had the same restrictions used in Experiment 1 
and 2, with the exception that at least two (instead of three) trials without a surprise stimulus had to occur between any two surprise trials.

\section{Results and Discussion}

Participants whose baseline probe detection rate was low $\left(d^{\prime}<0.5\right)$ were excluded from further analyses (Table 3). We investigated effects within each group first, and then compared across groups to understand the effects of surprise stimulus heterogeneity and density.

\section{Probe Reports Within Each Group}

For visualization purposes, the average probe report rate for each of the four groups was calculated for each trial type and block (Figure 3B-E). For hits, the main effect of trial type was significant in each group (Table 4), demonstrating that surprise stimuli impaired performance. Three of the four groups also had significant Trial Type $\mathrm{x}$ Time interactions (Table 4). The groups with the same surprise stimuli in each surprise trial evidenced clear effect habituation across repeated presentations: Hit rates significantly increased over time (across blocks) for surprise trials (Same 10\% group: $p=.001$, Same 20\% group: $p=.005$ ), whereas the hit rate for probe-only trials either decreased $(p=.020)$ or remained unchanged $(p=.55)$. Habituation effects were less clear for the varying groups. The Varying $10 \%$ group showed non-significant changes across time for surprise trials $(p=.079)$ and probe-only trials $(p=.29)$. The Trial Type $\mathrm{x}$ Time interaction was not significant for the Varying $20 \%$ group, but the main effect of time was significant.

Table 4. Statistical results for GLMMs assessing probe hit rates in Experiment 3 as a function of time and trial type. Significant effects are indicated in bold.

Same $10 \% \quad$ Same $20 \% \quad$ Varying $10 \% \quad$ Varying $20 \%$




\begin{tabular}{lcccccccc} 
& $\chi^{2}(1)$ & $p$ & $\chi^{2}(1)$ & $p$ & $\chi^{2}(1)$ & $p$ & $\chi^{2}(1)$ & $p$ \\
\hline Trial type & $\mathbf{3 8 . 5 8}$ & $<.001$ & $\mathbf{2 3 . 6 2}$ & $<.001$ & $\mathbf{6 3 . 4 9}$ & $<.001$ & $\mathbf{1 0 3 . 6 7}$ & $<.001$ \\
Time & 1.04 & .31 & 0.37 & .54 & 0.32 & .57 & $\mathbf{5 . 0 1}$ & $\mathbf{. 0 2 5}$ \\
\hline Trial Type x Time & $\mathbf{1 9 . 0 7}$ & $<.001$ & $\mathbf{9 . 3 1}$ & $\mathbf{. 0 0 2}$ & $\mathbf{6 . 0 6}$ & $\mathbf{. 0 1 4}$ & 3.65 & .056
\end{tabular}

Probe detection performance for each trial type was similar across groups (Figure 3). Notably, accuracy for the first surprise trial (which always contained a probe item) was similar across the four groups (Same 10\%: 48\%; Same 20\%: 47\%; Varying 10\%: 57\%; Varying 20\%: 60\%). These values did not significantly differ (Cochran $\mathrm{Q}(3)=2.97, p=.40)$.

As we had found in Experiments 1 and 2, the false alarm rate either decreased over time (Same 20\%: $\chi^{2}(1)=6.86, p=009$; Varying 20\%: $\left.\chi^{2}(1)=20.90, p<.001\right)$ or did not significantly change. (The Same GLMMs included random intercepts only.) A significant Trial Type $\mathrm{x}$ Time interaction for Same $10 \%\left(\chi^{2}(1)=7.14, p=.008\right)$ was due to a significant decrease for surprise trials $(p=.005 ; p=1.00$ otherwise). The higher false alarm rate for surprise trials in the Same $20 \%$ group $\left(\chi^{2}(1)=13.89, p<.001\right)$ was both numerically small and apparently an isolated oddity. No other effects were significant $\left(\chi^{2}(1) \mathrm{s}<2.14, p \mathrm{~s}>.14\right)$. Overall, the false alarm rate here mirrored our lab-based results. Our surprise effects were not due to response bias or participants mistakenly reporting the surprise stimulus as the probe, even with online experimentation.

\section{Formal Comparisons Across Groups}

Our analyses showed robust surprise-induced deafness effects within each group, though the results also suggested differences across them. To test these observations statistically, we constructed and fit a GLMM with heterogeneity (same, varying) and density 
$(10 \%, 20 \%)$ as between-subject variables, and time (continuous) and trial type (probe-only,

Lag 3 surprise) as within-subject variables.

Table 5. Statistical results for a GLMM assessing probe hit rates as a function of time, trial type, heterogeneity, and density. Significant effects are indicated in bold.

\begin{tabular}{|c|c|c|}
\hline & $\chi^{2}(1)$ & $p$ \\
\hline Time & 3.05 & .08 \\
\hline Trial type & 216.81 & $<.001$ \\
\hline Heterogeneity & 0.77 & .38 \\
\hline Density & 2.76 & .10 \\
\hline Trial Type $\times$ Time & 32.56 & $<.001$ \\
\hline Heterogeneity $\times$ Time & 1.33 & .25 \\
\hline Heterogeneity $\times$ Trial Type & 6.71 & .01 \\
\hline Density $\times$ Time & 0.86 & .35 \\
\hline Density $\times$ Trial Type & 2.46 & .12 \\
\hline Heterogeneity $\times$ Density & 0.03 & .85 \\
\hline Heterogeneity $\times$ Trial Type $\times$ Time & 3.45 & .063 \\
\hline Density $\times$ Trial Type $\times$ Time & 1.89 & .17 \\
\hline Heterogeneity $\times$ Density $\times$ Time & 1.63 & .20 \\
\hline
\end{tabular}




$\begin{array}{lll}\text { Heterogeneity } \times \text { Density } \times \text { Trial Type } & 0.29 & .59 \\ \text { Heterogeneity } \times \text { Density } \times \text { Trial Type } \times \text { Time } & 0.09 & .77\end{array}$

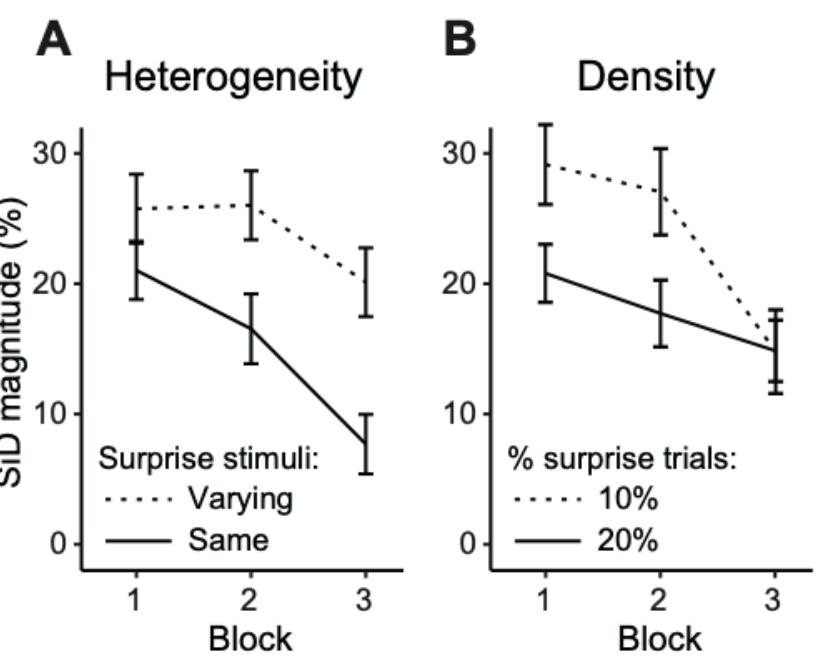

Figure 4. Summary of effects from the heterogeneity and density manipulations in Experiment 3. Mean SiD magnitude aggregated by the (A) heterogeneity or (B) density groups as a function of time. For the latter, note that there are twice as many surprise trials per block in the $20 \%$ Density condition. Error bars represent \pm 1 standard error of the mean (SEM).

\section{Surprise Stimulus Heterogeneity}

For visualization purposes, "SiD magnitude" was calculated by subtracting the surprise trials' hit rate from the probe-only trials' hit rate (Figure 4A). The GLMM analysis revealed a significant interaction of Heterogeneity x Trial Type (Table 5), indicative of greater $\mathrm{SiD}$ when surprise stimuli varied across trials $\left(M_{\text {same }}=16.45 \%, S D_{\text {same }}=30.76 \% ; M_{\text {varying }}=\right.$ $23.96 \%, S D_{\text {varying }}=29.71 \%$ ). Follow-up analyses showed that surprise trials were affected by the heterogeneity manipulation $(p=.030)$, whereas probe-only trials were not $(p=.92)$. The 
significant Trial Type $\mathrm{x}$ Time interaction (Table 5) reflected both an improvement in surprise trial performance $(p<.001)$ and a decrease in probe-only trial performance $(p<.001$; see Figure 3).

Varying the stimuli across surprise trials led to larger deficits, despite the varying group beginning with numerically smaller effects in the first surprise trial. Although there was some suggestion of slower effect habituation with varying surprise stimuli (see Figure 4A), the relevant comparisons $($ Heterogeneity $\times$ Time, Heterogeneity $\times$ Trial Type $\times$ Time; see Table 5) were not statistically significant. Overall, the results are consistent with surprises being distracting because of the information they contain. Even as individuals learned that the task involved unexpected events, the surprise stimuli's novel identities caused a reallocation of attention to the detriment of probe detection. Although this reallocation contains stimulusdriven components, it may also involve goal-directed or other forms of attentional control, an idea to which we return in the General Discussion.

\section{Surprise Stimulus Density}

For visualization, the timecourse of $\mathrm{SiD}$ magnitude was compared across the $10 \%$ and $20 \%$ groups (Figure 4B). Neither the main effect of density nor any of its interactions were significant (Table 5). Although rarer surprise stimuli caused a non-significantly greater SiD effect, the effect habituation differences were, if anything, opposite to our expectations. The $10 \%$ Density groups habituated more rapidly even though the time variable did not account for the different number of surprise trials across density groups. When we fit an alternative model with slopes representing the degree of change from one surprise trial to the next (instead of change across the entire session), we found similar results with virtually identical patterns of significance. The one exception was a significant Density $\times$ Trial Type $\times$ Time interaction $\left(\chi^{2}(1)=9.19, p=.002\right)$, confirming faster habituation of surprise effects in the $10 \%$ Density groups (see Figure 4B). Future experiments with stronger density 
manipulations could address this unexpected result as well as the unconvincingly null main effects.

\section{Comparison of Lab-Based and Online Results}

The lab-based (Experiment 2) and online (Experiment 3, Same 10\% group) experimental results were compared in a single GLMM by adding a between-groups variable of experiment. Neither the main effect of experiment $\left(\chi^{2}(1)=0.12, p=.73\right)$, the Time $\mathrm{x}$ Experiment interaction $\left(\chi^{2}(1)=0.52, p=.47\right)$, nor the Time $\mathrm{x}$ Trial Type $\mathrm{x}$ Experiment interaction $\left(\chi^{2}(1)=0.72, p=.40\right)$ was significant. The Trial Type $\mathrm{x}$ Experiment interaction $\left(\chi^{2}(1)=6.21, p=.013\right)$ was significant, but performance was not significantly lower in Experiment 3 during either surprise trials $(p=.083)$ or probe-only trials $(p=.56)$. At worst, the significant interaction suggests larger $\mathrm{SiD}$ effects in the online version (Experiment 3; see Figures $3 \mathrm{~A}$ and $3 \mathrm{~B}$ ). Note also that $\mathrm{SiD}$ magnitude in Experiment 3 (Same 10\% group) was similar to that observed in Experiment 1A (Figure 2). For false alarms, the main effect of time $\left(\chi^{2}(1)=7.16, p=.007\right)$, the main effect of trial type $\left(\chi^{2}(1)=6.09, p=.014\right)$, and the interaction of Time $\mathrm{x}$ Trial Type $\mathrm{x}$ Experiment $\left(\chi^{2}(1)=7.38, p=.007\right)$ were significant (all other effects: $\left.\chi^{2}(1)<1.93, p s>.16\right)$. The three-way interaction was driven by Experiment 2's significant decrease in false alarms in trials without a surprise stimulus over time $(p=.006)$, an effect absent from Experiment $3(p=1.00)$. Taken together, these results suggest that SiD effects are largely comparable across lab-based and online experiments.

\section{Experiment 4: Behavioral relevance of surprise stimuli}

For the tone tasks in Experiments 1-3, each surprise stimulus was a spoken letter (Experiments 1-3) or an environmental sound (Experiment 3). Surprise stimuli from these categories may capture attention more easily than other types, as they are broadly relevant to behavior even as they are irrelevant to the specific task (Egeth \& Yantis, 1997). Novel 
environmental sounds and tone deviants evoke partially dissociable electrophysiological and behavioral effects as well (Escera et al., 1998). Thus, it is possible that the tone surprise stimuli contributed to the letter task's unclear results (Experiment 1B). In Experiment 4, we tested whether different categories of surprise stimuli (constructed sine-wave tones or meaningful auditory objects) would capture attention due to their deviance from expectation and thereby produce comparable $\mathrm{SiD}$ effects. Conversely, if broad behavioral relevance of the surprise stimuli is an important factor in inducing $\mathrm{SiD}$, we might expect reduced or even absent effects with these less meaningful tones. A secondary aim was to test whether SiD would still be observed when baseline detection performance was high for virtually all participants.

\section{Method}

The current experiment was identical to the Varying 20\% condition of Experiment 3 with the following exceptions. Thirty-nine participants (14 females, 25 males, mean age $=$ 28.74 years, age range $=22-42$ ) completed the task; we aimed for a sample size twice that of Experiment 2, but had a data recording error for one individual. The experimental task included 12 Lag 3 surprise trials, each with a different surprise stimulus. Six surprise stimuli were auditory objects drawn from Experiments 1-3: a spoken letter "i", a spoken digit "one", an alarm, a cough, a dog barking, and a hiccup. Six other surprise stimuli were complex tones, each comprised of five log-related frequencies from the following ranges: 455-792, 794-1260, 909-1583, 944-2381, 1349-2142, and 1819-3167 Hz. As in Experiment 2, all stimuli were envelope-normalized, which yielded subjectively similar intensities. After this equilibration, the probe was set to $45 \%$ and distractors were set to $30 \%$ of the surprise stimuli's volume. Finally, the three highest-pitch distractors were removed to make probe detection slightly easier.

Before beginning the main experiment, participants completed 8 practice trials at a 
slower speed $(\mathrm{SOA}=130 \mathrm{~ms})$ and 8 practice trials at normal speed $(\mathrm{SOA}=120 \mathrm{~ms})$. Practice blocks contained feedback but no surprise stimuli. For the main experiment, participants completed two blocks of 30 trials each. Participants reported whether the probe was present or absent when prompted at the end of each trial.

\section{Results and Discussion}

Four participants $(10 \%$ of the sample) had responses that were indistinguishable from random responding. Data from these individuals were removed, leaving 35 individuals in the final sample. Given the brevity of the experiment and its similarity to the Varying $20 \%$ condition in Experiment 3, we focused our analysis on overall surprise effects. These effects were evidenced by significantly lower performance during Lag 3 surprise trials (mean = $83.49 \%, S D=27.27 \%$ ) compared to probe-only trials (mean $=98.25 \%, S D=3.37 \%$; Wilcoxon signed-ranks test: $p=.002$ ). In contrast, the hit rate following auditory objects (mean $=82.86 \%, S D=29.16 \%)$ was similar to the hit rate following complex tones $($ mean $=$ $84.29 \%, S D=32.70 \% ; p=1.00$ ). The false alarm rate was slightly but significantly higher for no-probe trials $($ mean $=8.81 \%, S D=12.77 \%$ ) compared to surprise-only trials (mean $=$ $4.76 \%, S D=16.46 \% ; p=.04)$. Importantly, this rate did not differ across auditory object $($ mean $=4.29 \%, S D=14.20 \%)$ and complex tone $($ mean $=5.71 \%, S D=23.55 \%)$ surprise stimuli $(p=1.00)$.

Both real-world, recognizable sounds and artificially-generated tones yielded similar SiD results. This finding is consistent with other auditory experiments involving capture and distraction, in which deviant tones are frequently used to evoke brain responses and induce behavioral costs (Friedman et al., 2001). The experiment also showed that SiD could be induced even when baseline probe detection was virtually perfect.

\section{Experiment 5: Capture in singleton detection mode?}


In Experiments 1-4, we showed that relatively unexpected and rare stimuli can capture attention, thereby impairing probe detection. We argued that the surprise stimuli disrupted the task despite being irrelevant to searching for the probe item. Another possibility, however, is that participants adopt a "singleton detection mode" to identify the probe item (Bacon \& Egeth, 1994; Egeth \& Yantis, 1997). As the probe has a significantly higher pitch than the distractor items, participants may simply listen for a deviant sound. If they do so, the surprise stimulus may capture attention because it, like the probe, is a singleton (Dalton \& Lavie, 2004).

Various features of our experiments suggest that participants were not using "singleton detection mode", at least not exclusively. The disruptive effects of a given surprise stimulus were sensitive to its frequency of appearance and relative novelty, with effects habituating across multiple presentations (Experiments 1-3). Nevertheless, we sought a more direct test. We examined whether $\mathrm{SiD}$ would occur when the probe was defined by a specific intermediate pitch amongst other distractor tones. A singleton strategy would likely not work in such circumstances (Bacon \& Egeth, 1994).

\section{Method}

The current experiment was identical to the Same 20\% condition of Experiment 3 with the following exceptions. Forty participants ( 25 males, 15 females, mean age $=29.23$ years, age range $=22-51$ ) completed the task. The stimuli were five pure tones of log-related frequencies, specifically 396, 629, 1000, 1587, and $2519 \mathrm{~Hz}$. The $1000 \mathrm{~Hz}$ tone served as the probe. The number of tones was decreased so that the task remained feasible for participants. To help them further, they completed 8 practice trials at a slower speed $(\mathrm{SOA}=130 \mathrm{~ms})$ and 8 practice trials at normal speed $(\mathrm{SOA}=120 \mathrm{~ms})$ before beginning the experimental trials. Practice blocks contained feedback but no surprise stimuli.

Two types of surprise stimuli were generated from distractor tones of either lower or 
higher frequencies than the probe tone (i.e., $1000 \mathrm{~Hz}$ ), with care taken to ensure they would not be confused for the probe. The "low" surprise stimulus was a tone gliding from 396 to $629 \mathrm{~Hz}$, whereas the "high" surprise stimulus was a tone gliding from 1587 to $2519 \mathrm{~Hz}$. Participants were randomly assigned to either the low or high surprise stimulus groups. Stimulus power was equalized across all sounds as in Experiment 4.
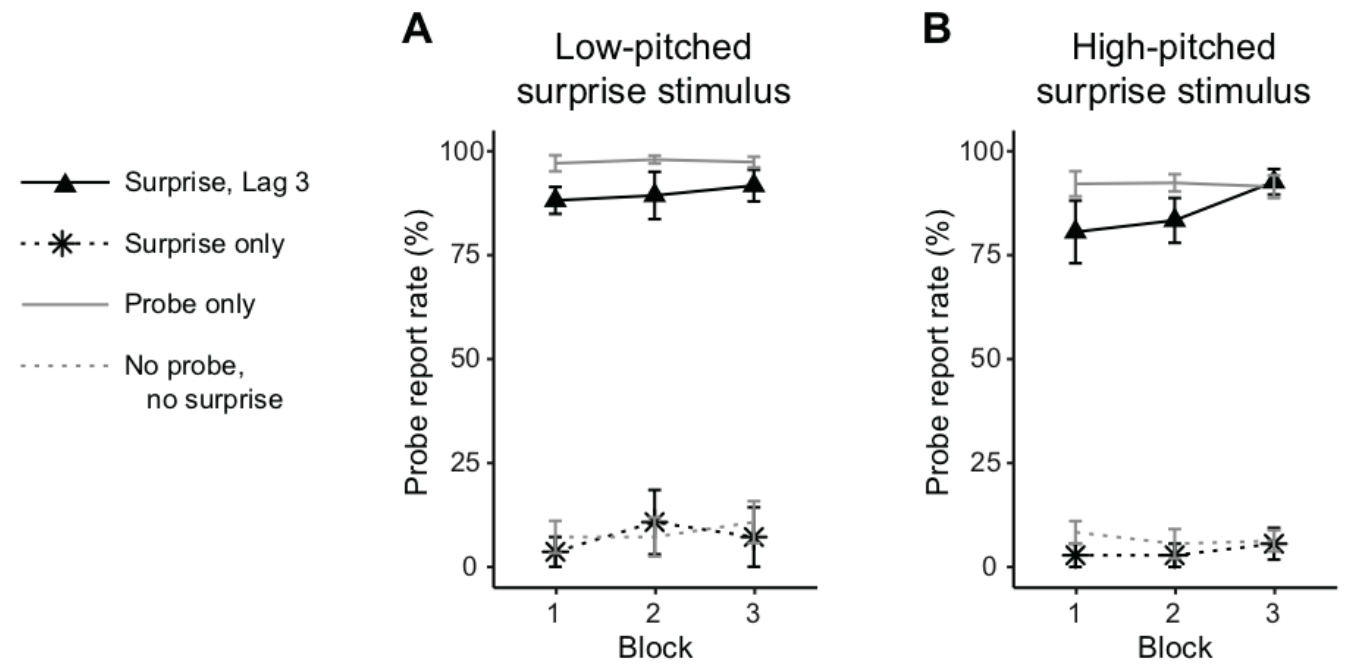

Figure 5. Mean probe report results for Experiment 5 presented by block and trial type for the low (A) and high (B) surprise groups. Reports in the absence of a probe represents false alarms.

\section{Results and Discussion}

Participants whose performance was indistinguishable from random responding $(\mathrm{n}=$ 8, 20\%) were excluded from further analyses. 32 individuals were thus included in the final sample, 14 in the low surprise stimulus group and 18 in high group. For visualization purposes, the average probe report rate was calculated by block for each trial type and surprise stimulus group (Figure 5). The data were analyzed using GLMMs with a between- 
groups variable of surprise stimulus group (low or high; categorical) and within-subject variables of time (continuous) and trial type (probe-only, Lag 3 surprise; categorical). For hits, the significant main effect of trial type $\left(\chi^{2}(1)=21.22, p<.001\right)$ indicated reduced performance for Lag 3 surprise trials (Figure 5). A significant Time x Trial Type interaction $\left(\chi^{2}(1)=8.24, p=.004\right)$ was due to $\mathrm{SiD}$ habituation; across time, surprise trial performance improved $(p=.005)$, whereas probe-only performance did not significantly change $(p=$ 1.00). Follow-up Wilcoxon signed-rank tests showed that Lag 3 surprise performance was lower only during the first block ( $p=.007$; subsequent blocks: $p$ s $>.06)$. Indeed, the hit rate for the very first surprise trial ( 21 of $32 ; 66 \%$ ) was lower than the hit rate for the preceding probe-only trial (28 of $32,88 \%$; directional proportion test: $\chi^{2}(1)=3.13, p=.04$ ). There was also a significant Trial Type $\mathrm{x}$ Surprise Stimulus Group interaction $\left(\chi^{2}(1)=4.10, p=.043\right)$, though surprise effects were significant for each group $(p s<.018)$ and comparable across them $\left(M_{\text {low }}=7.74 \%, S D_{\text {low }}=10.01 \% ; M_{\text {high }}=6.48 \%, S D_{\text {high }}=11.26 \%\right.$; comparison across groups: $p=.74)$. No other main effects or interactions were significant for hits $\left(\chi^{2} \mathrm{~s}<1.82, p \mathrm{~s}\right.$ $>$.18). For false alarms, there were no significant main effects or interactions $\left(\chi^{2} \mathrm{~s}<1.12, p \mathrm{~s}\right.$ $>$.29; the model included random intercepts only).

In sum, the current experiment shows that surprise-induced deafness, both the deficit itself and its characteristic pattern of habituation, is still present when the probe is not a singleton amongst the distractor items. We conclude that SiD depends critically on stimulusdriven attentional capture by a salient task-irrelevant item. Other factors, including goaldirected attentional influences, may contribute to the deficit as well. We explore this idea in a final pair of experiments and in the General Discussion.

\section{Experiment 6: Replicating and extending the auditory attentional blink (AAB)}

In our surprise-induced deafness paradigms, participants search for a probe item amongst distractors, and failure to detect that probe is the critical measure. Similar aspects are 
central to the auditory attentional blink (AAB) as well (Arnell \& Jolicoeur, 1999; Mondor, 1998). Despite their general similarity, the two paradigms have a crucial difference: Whereas $\mathrm{SiD}$ is caused by an unexpected and task-irrelevant stimulus, the AAB is caused by processing a target item that precedes the probe. The AAB substantially-sometimes completely_attenuates when participants are told to ignore the target stimulus (Arnell \& Jolicoeur, 1999; Horváth \& Burgyán, 2011). As such, the AAB appears to be caused by goaldirected attentional processing of the target, not stimulus-driven attentional capture (Arnell \& Jenkins, 2004; Arnell \& Jolicoeur, 1999; Martens et al., 2009, 2010; Mondor, 1998; Shen \& Mondor, 2006; Vachon et al., 2009; Vachon \& Tremblay, 2005, 2006).

In the current experiment, we tested whether an $\mathrm{AAB}$ would be found with a paradigm adapted from our $\mathrm{SiD}$ experiments. This approach allowed us to compare the two deficits when using highly similar procedures and stimuli. For example, three complex tones that were used as surprise stimuli in Experiment 4 were here used as targets. We could therefore test the effects of the same stimuli when they played different roles. The experiment also allowed us to prepare for Experiment 7, in which we used an individual differences approach to further investigate the relationship of $\mathrm{SiD}$ and the $\mathrm{AAB}$.

\section{Method}

\section{Participants}

Forty-three NUS undergraduates with normal hearing participated for course credit. We used a target-probe AAB design for similarity to our SiD paradigm. Participants were randomly allocated to either the experimental group (detect and report both the target and the probe) or to the control group (detect and report only the probe). Three participants in the former group (age and gender not recorded) found the task too difficult during the practice trials and elected not to continue. 20 individuals in each group completed the task (experimental: 14 females, 6 males, mean age $=20.30$, age range $=18-24$; control: 11 
females, 9 males, mean age $=21.7$, age range $=19-25$ )

\section{Stimuli and Apparatus}

Distractor stimuli were pure tones of log-related frequencies ranging from 639 to $2911 \mathrm{~Hz}$ (Figure 6A). The same stimulus set (save $3175 \mathrm{~Hz}$ ) was used for Experiments 1-3. The probe was again a $4000 \mathrm{~Hz}$ pure tone. Three target stimuli were constructed from five log-related frequencies: 455-792 Hz (“low”), 909-1583 Hz (“middle"), and 1819-3167 Hz ("high"). Note that these stimuli were used as surprise stimuli in Experiment 4, thereby allowing us to compare the effects of the same stimuli playing different roles. As in Experiment 2, all stimuli were envelope-normalized. The experiment was conducted in the laboratory, with PsychoPy software version 1.81 .03 (Peirce, 2007) and a Dell computer (OPTIPLEX 990) used for stimulus presentation and data collection. Sounds were presented binaurally through TDK headphones (ST 100) with an approximate maximum volume of 70 dB. Participants made their responses on a standard computer keyboard. 
A Auditory attentional blink paradigm

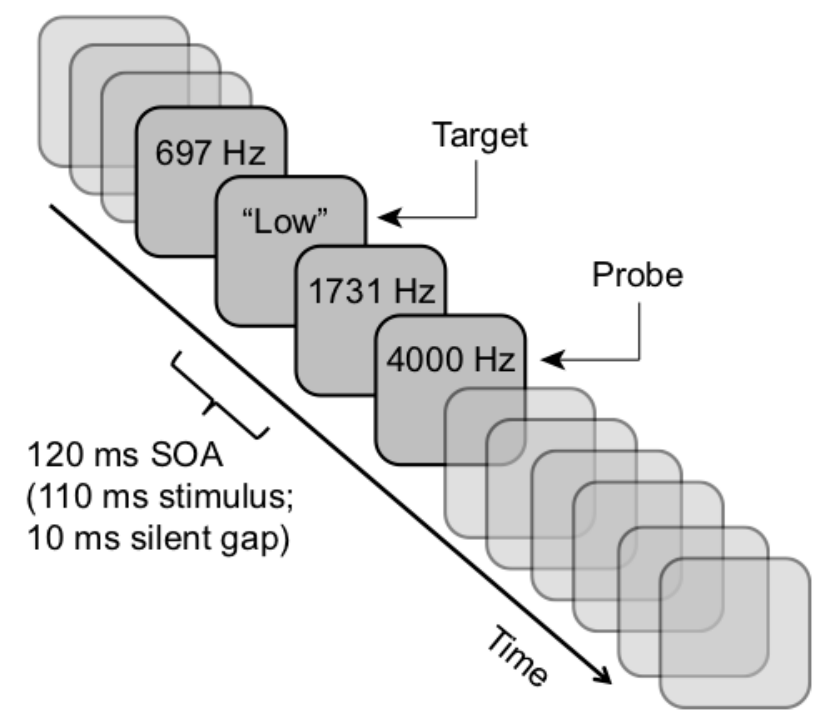

B AAB paradigm with surprise stimuli

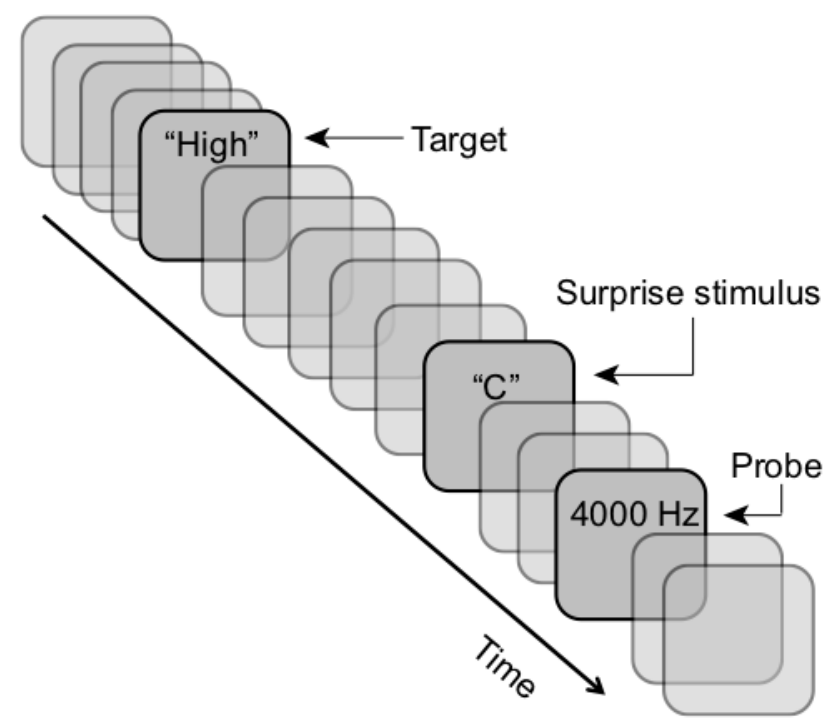

Figure 6. A) Stimulus sequence for trials in the auditory attentional blink (AAB) paradigm in Experiment 6. A Lag 2 trial is shown. B) Stimulus sequence for trials in the AAB-SiD hybrid paradigm in Experiment 7. A Lag 3 surprise trial is shown. Note that surprise trials with the probe were also Lag $9 \mathrm{AAB}$ trials (though they were analyzed separately).

\section{Task Procedures}

Before each trial, the task-relevant tones (target and probe for the experimental 
group, only the probe for the control group) were played to refresh participants' auditory memory. Participants could play these demo tones as many times as they chose. A fixation cross was then displayed until the participant pressed any key to begin a rapid auditory presentation (RAP) of 30 tones. One of the three targets was presented during each trial, followed by the probe on $75 \%$ of trials. When present, the probe was presented after $17-27$ distractors and at Lag 1, 2, 3, 5, or 8 after the target. Participants reported the probe as soon as it was detected, thereby giving us a secondary measure of performance impairment, as in Experiment 1. If participants did not make a response during the RAP, they were prompted by a question (“Probe?”) $300 \mathrm{~ms}$ after the RAP's conclusion. Participants pressed the '<' key for "present" and the '>' key for "absent". Participants in the experimental group were then prompted to indicate which target was presented (“Target?") by pressing the ' 1 ', ' 2 ', or ' 3 ' key. For probe detection, both accuracy and speed were emphasized ("Respond as quickly as it is possible (but not at the expense of accuracy) using your right hand"), whereas speed was not emphasized for target discrimination.

Participants completed 240 trials presented in 6 blocks; each block contained 6 trials for each target-probe lag and 10 no-probe trials. Participants were encouraged to take brief breaks between blocks. Before beginning the main task, participants in the experimental group practiced at least three phases of 6 trials each: target discrimination, probe detection, and both tasks (Lag 8 only). Participants in the control group practiced only probe detection. For each phase of practice, participants had to reach an accuracy of $66 \%$ before advancing to the next phase.

\section{Statistical Approach}

For the auditory attentional blink in Experiments 6 and 7, the key variables of were all categorical. Therefore, we assessed their effects on response rates and times using standard repeated-measures ANOVAs using ezANOVA (version 4.4-0) (Lawrence, 2016). 
Greenhouse-Geisser correction was applied when the assumption of sphericity was found to have been violated. For such cases and when variances were unequal in independent-samples t-tests, we report uncorrected degrees of freedom and corrected F-values and p-values. Effect sizes for significant results are reported as generalized eta-squared values (denoted as " $\eta G^{2}$ "), which are presented after the corresponding p-values.

\section{Results and Discussion}

No participants were removed for low probe detection performance based on the criteria described in Experiment 1. Two participants, however, had target discrimination accuracy that was not distinguishable from chance performance $(<40 \%)$. These individuals were removed from the sample, leaving 18 in the experimental group.

\section{Target Discrimination}

Average target report accuracy was $69.88 \%(S D=12.58 \%)$, indicating a challenging task. This rate did not vary significantly by lag (repeated-measures ANOVA: $F(4,68)$

$=1.18, p=.33$ ). Furthermore, target performance did not significantly differ across trials with a probe $($ mean $=69.18 \%, S D=12.91 \%)$ compared to those without one $($ mean $=71.92 \%, S D$ $=13.13 \%$; paired-samples t-test: $t(17)=1.59, p=.13)$.

A

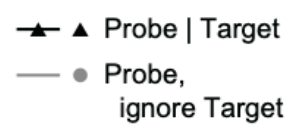

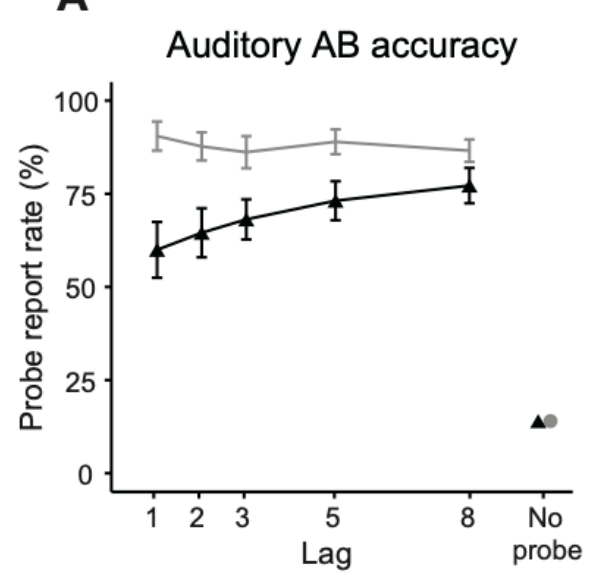

B

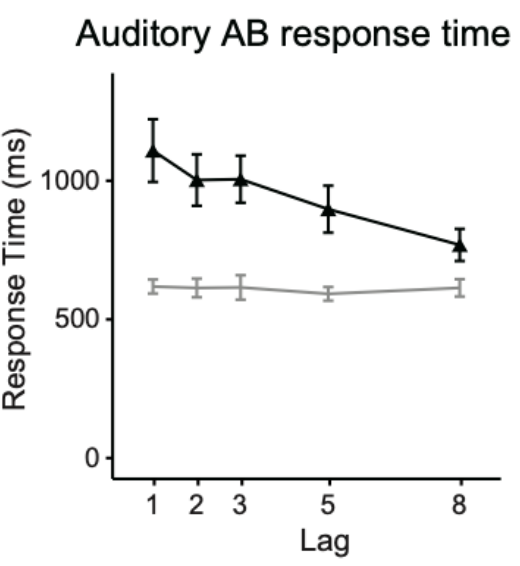


Figure 7. Probe performance results for Experiment 6. A) Mean probe report by lag for the experimental (probe | target) and control (probe, ignore target) groups. For the former, probe report was calculated contingent on correct target identification. The report rate for "no probe" represents the false alarm rate. B) Mean probe response times by lag for the experimental and control groups. Trials for the former were again contingent on correct target identification.

\section{Probe Report}

For the experimental group, probe report rates were calculated from trials with correct target report (probe $\mid$ target). Mean report rates were submitted to a mixed $5 \times 2$ twoway ANOVA with lag as a within-subject variable and group as a between-subject variable. Both the main effects of $\operatorname{lag}\left(F(4,144)=3.32, p=.030, \eta_{G}{ }^{2}=.015\right)$ and group $(F(1$, $\left.36)=9.25, p=.004, \eta_{G}^{2}=.18\right)$ were significant, as was their interaction $(F(4,144)$ $=6.68, p=.001, \eta_{G}^{2}=.03$ ). Performance improved with increasing lag for the experimental group (repeated-measures ANOVA: $\left(F(4,68)=5.47, p<.001, \eta_{G}{ }^{2}=.06\right)$, but not for the control group $(F(4,76)=1.84, p=.13)$. Compared to the control group, performance in the experimental group was significantly lower for Lags 1 and 2 (independent samples t-tests: $p$ s $<.026)$, but not for Lags 3, 5, or $8(p \mathrm{~s}<.079)$. The mean false alarm rates did not significantly differ across groups $(t(36)=0.04, p=.84)$.

These results are consistent with the presence of an auditory attentional blink. The effect lasted for several hundred milliseconds within each trial, in line with previous AAB reports (Mondor, 1998; Vachon \& Tremblay, 2005) and grossly similar to the timecourse of SiD. Unlike in SiD, however, goal-directed processing of a target caused the AAB deficit. Participants in the control group, who ignored target stimuli, evidenced no lag-dependent probe detection deficits. These same target stimuli, however, induced deficits through 
stimulus-driven attentional capture when they were relatively unexpected and rare, as evidenced in Experiment 4.

\section{Probe Reaction Times}

For the experimental group, only trials with correct target reports were included for the analysis. A GLMM with variables of lag and group was fitted with a gamma error distribution (log-linked) and random intercepts. The main effect of group $\left(\chi^{2}(1)=172.11, p\right.$ $<.001)$ and the Lag x Group interaction $\left(\chi^{2}(4)=14.19, p=.007\right)$ were both significant, while the main effect of lag was not $\left(\chi^{2}(4)=7.85, p=.10\right)$. For each lag, reaction times were longer in the experimental group compared to the control group $(p \mathrm{~s}<.003)$. In the experimental group, reaction times were significantly longer for Lag 8 compared to Lags 1, 2, or 3 $(p s<.024)$ and not significantly different between any other lag pairs $(p s>.11)$. No significant pairwise differences were observed for the control group $(p s=1.00)$. Although not necessarily indicative of an auditory attentional blink per se (Arnell \& Jolicoeur, 1999; Ruthruff \& Pashler, 2001), these reaction time results reflect a cost to attending to the first target. Consistent with the probe hit rate results presented above, the first target only imposed this cost when it was task-relevant.

\section{Experiment 7: The relationship between $\mathrm{SiD}$ and the $\mathrm{AAB}$}

In Experiment 6, we successfully demonstrated an auditory attentional blink (AAB) using a paradigm adapted from our $\mathrm{SiD}$ experiments. In this final experiment, we compared the two effects directly. Despite their similarity in procedure and results, $\mathrm{SiD}$ and the $\mathrm{AAB}$ are associated with different failures of attentional control. SiD is induced by a surprise stimulus redirecting attention in a stimulus-driven fashion, whereas the AAB is caused by goal-directed attentional processing of a target item. Our experimental results clearly reflect this distinction. An AAB was not triggered by stimulus-driven attentional capture, whereas $\mathrm{SiD}$ was found even for the very first surprise trial with stimuli that contained no target- 
defining features.

Nevertheless, the two deficits may share some common mechanisms. One possibility is that the set of processes involved in goal-directed and stimulus-driven attentional control overlap. The overlap may allow the two forms of attentional control to interact, supporting coherent behavior. It may also indicate the presence of neurocognitive processes, such as inhibition or stimulus disengagement (Luck et al., 2020), that are common to both the AAB and $\mathrm{SiD}$ as well as to both forms of attentional control. Another possibility is that the SiD deficit is modulated by goal-directed attentional control processes that are also relevant to the $\mathrm{AAB}$ deficit. For example, both paradigms require participants to shift their attention to probe detection following either distraction $(\mathrm{SiD})$ or processing of a target item $(\mathrm{AAB})$. A more rapid or effective shift would presumably reduce either deficit.

In the current experiment, we used an individual differences approach to better understand the relationship between $\mathrm{SiD}$ and the $\mathrm{AAB}$. If the deficit magnitudes are correlated across participants, we would infer that they reflect some shared cognitive mechanisms; the magnitude of the correlation would be related to the degree of the shared mechanisms. This approach and reasoning have been used to show that attentional blinks draw on separate resources across modalities (Liaw et al., 2020; Martens et al., 2009, 2010), and that different types of visual ABs (broadly, those with probe detection versus target discrimination for the second item) have partially dissociable causes (Dale et al., 2013; Kelly \& Dux, 2011). To keep the experimental session manageably brief, we estimated the two deficits' magnitudes within a single paradigm. In addition, we included a relatively small control group who ignored the targets (see Experiment 6). This control group allowed us to confirm that ignored targets would not produce probe detection deficits with our stimuli and procedure.

\section{Method}


The current experiment combined the $\mathrm{AAB}$ and SiD paradigms, adapting methods from Experiment 6 and Experiment 3 (Varying 20\% condition) with additional modifications. For clarity, the key design elements are reproduced below.

\section{Participants}

Two hundred participants (115 males, 81 females, 4 gender not reported; mean age = 30.74 years, age range $=20-61,2$ age not reported) completed the experiment on Amazon Mechanical Turk (AMT). This sample size allowed us to detect small correlation effects $(r=$ 0.2 ) with $80 \%$ power, which we deemed necessary given that even different forms of the visual attentional blink paradigm correlate at only a moderate level $(r=0.4)$ (Dale et al., 2013; Kelly \& Dux, 2011; MacLean \& Arnell, 2012). Due to a data collection error, the data from one participant could not be used. In addition to this experimental group, 25 participants $(13$ males, 12 females, mean age $=30.28$ years, age range $=24-39)$ completed a control version of the experiment, in which the target was ignored. This smaller control group allowed us to test whether the targets captured attention in a stimulus-driven fashion, and whether targets affected SiD itself. All participants received \$3 USD for the study, which lasted approximately 50-60 minutes.

\section{Stimuli and Apparatus}

As in Experiment 6, distractor stimuli were pure tones of log-related frequencies ranging from 639 to $2911 \mathrm{~Hz}$, and the probe was a $4000 \mathrm{~Hz}$ pure tone. The two complex tone targets were constructed from five log-related pure tones, with ranges of 794-1260 and 1349$2142 \mathrm{~Hz}$. The number of target alternatives was reduced in an attempt to shorten the blink period, thereby more clearly separating within-blink probe detection performance from probe detection that was not affected by target processing (Shore et al., 2001). Twenty-four surprise stimuli from Experiments 1-5 were selected for the present experiment. The set consisted of spoken letters ('C', 'L', 'J', 'H'), spoken digits ('one', 'two'), and environmental sounds 
(alarm, balloon popping, car honking, cat meowing, cough, cowbell, dog barking, giggle, hiccup, hi-hat cymbal, light bulb breaking, mosquito buzzing, plunger, popping tongue, slide whistle, snare drum, sneeze, slurping). Widely-varying surprise stimuli were used in an effort to reduce SiD habituation, enabling us to obtain more robust effects and stable individual differences. After envelope-normalization, stimuli were further adjusted so that their amplitudes were $30 \%$ (distractors and surprise stimuli) or $45 \%$ (probe) of the targets' amplitudes.

\section{Task Procedure}

Before each trial, the target and probe stimuli were played in sequence to refresh participants' auditory memory. Participants could play the stimuli as many times as they chose. A fixation cross was then displayed until the participant pressed any key to begin a rapid auditory presentation (RAP) of 30 tones. One of two targets was presented during each trial, which was followed by the probe on $75 \%$ of trials. When present, the probe was presented after 17-27 RAP items at Lag 1, 2, 3, 5, 7, or 9 after to the target. During $20 \%$ of the trials, a surprise stimulus was played six items $(720 \mathrm{~ms})$ after the target (Figure 5B). At Lag 6, the surprise stimulus would be largely clear of the AAB window; pilot testing confirmed that the surprise stimulus was clearly audible (and reported to be distracting) in this position. $75 \%$ of the surprise trials contained a probe, which followed the surprise stimulus at Lag 3. This timing rendered the probe vulnerable to $\mathrm{SiD}$, while putting it outside the AAB window (Lag 9). At the conclusion of each RAP, participants were asked to identify the target by pressing ' $d$ ' (low) or ' $\mathrm{f}$ ' (high). They were next asked about the probe, responding with ' $\mathrm{j}$ ' for present and ' $\mathrm{k}$ ' for absent. All responses were unspeeded.

Participants completed 120 trials presented in 3 blocks. Each block contained exactly 6 surprise trials with a probe, 2 surprise trials without a probe, 24 standard trials with a probe (4 per Lag), and 8 standard trials without a probe. The trials were presented in a different 
random order for each block. Each participant experienced the same trial order, however, which reduced performance variability due to trial/stimulus differences. Before beginning the main task, participants were instructed to adjust the volume to their level of comfort and to use headphones if possible. They then completed 16 practice trials with feedback: 4 for target discrimination, 4 for probe detection, and 8 during which both tasks were performed. Targetonly practice trials contained no probes, whereas probe-only practice trials contained no targets. No surprise stimuli were presented during any practice trials.

The control group's version of the experiment was identical with the following exceptions. Participants were explicitly instructed to ignore the targets, and they were not queried about them at the conclusion of each trial. Only the probe sound was played before each trial, and only four practice trials (for probe detection) were completed.

\section{Analytical Approach}

Task performance for the $\mathrm{SiD}$ and $\mathrm{AAB}$ paradigm components was first assessed using the approaches from previous experiments. Data from participants with low probe detection or target discrimination performance removed as before. Preliminary analyses revealed that some participants evidenced probe detection that was consistently high, perhaps indicative of ceiling effects. As these individuals provided little behavioral variance of use, we removed their data for our correlation analyses. Participants were classified in this group if their probe detection hit rates, contingent upon correct target discrimination (probe $\mid$ target), were above $90 \%$ for both short lags (average of Lags 1 and 2) and long lags (average of Lags 7 and 9). Critically, we examined and report the correlations with these individuals included in the sample as well.

For correlation analyses, the magnitude of each effect for each participant was calculated using regression (MacLean \& Arnell, 2012). This approach controls for variation in baseline performance across participants. For $\mathrm{SiD}$, we regressed probe detection hit rates 
from Lag 9 trials against hit rates for surprise trials that contained a probe. The residuals were then saved and used for subsequent analyses (Dale et al., 2013). For the AAB, we regressed probe detection hit rates at long lags (Lags 7 and 9) against those at short lags (Lags 1 and 2), again saving the residual variability. For the $\mathrm{AAB}$ residuals, the input probe performance was calculated contingent on correct target identification; for SiD residuals, it was not. The metrics from which each set of residuals were calculated were chosen because they best reflected the deficits in question based on group-average performance and previous findings (Arnell \& Jolicoeur, 1999; Asplund, Todd, Snyder, Gilbert, \& Marois, 2010; Shen \& Mondor, 2008). For completeness, however, we considered two additional residual calculations. First, to compare the deficits at the same lag, we calculated AAB residuals by regressing probe detection at long lags (Lags 7 and 9) against Lag 3. Second, to compare the deficits by controlling for the same baseline performance, we calculated SiD residuals by regressing probe detection at long lags (Lags 7 and 9, contingent on correct target discrimination) against surprise trials that contained a probe.

The residuals for each deficit were used in two different ways. First, we calculated internal-consistency reliability scores of our effect measures using a split-half approach. The relevant trials for each metric component (e.g., Lag 7 and 9 trials, contingent on correct target discrimination) were identified and then split into odd or even trials according to their order of appearance. Residuals were calculated from each set of metric components, after which they were correlated across odd and even trials. Second, we calculated Pearson correlations amongst target discrimination accuracy, probe detection hit rates (not contingent on correct target discrimination), $\mathrm{SiD}$ magnitude (residuals), and $\mathrm{AAB}$ magnitude (residuals).

\section{Results and Discussion}

In the experimental group, 55 participants ( $28 \%$ of the sample) had probe detection performance below $d^{\prime}=0.5$. An additional 14 participants $(10 \%$ of the remaining sample $)$ had 
target discrimination accuracy below $60 \%$, which was indistinguishable from chance. The data from these participants were not included in subsequent analyses, leaving 130 participants in the final sample. Five participants ( $20 \%$ of the sample) in the control group had low probe detection performance. Their data were removed, leaving 20 participants in the final sample.

\section{SiD: Probe Reports}

In addition to the surprise trials themselves, standard Lag 9 and no-probe trials were included for the $\mathrm{SiD}$ analyses. The former provided a baseline for probe hit rates, whereas the latter provided the same for false alarms. For visualization, the mean hit and false alarm rates were calculated separately for each block (Figure 8A). For the experimental group, the main effect of trial type for hits was significant $\left(\chi^{2}(1)=46.20, p<.001\right)$, reflecting lower performance for surprise trials. Neither the main effect of time nor the Time $\mathrm{x}$ Trial Type interaction were significant $(p s>.19)$. Follow-up tests found that the surprise trial hit rate was lower than probe-only performance in each block (Wilcoxon signed ranks tests, $p$ $<.001)$. For false alarms, the Time $\mathrm{x}$ Trial Type interaction was not significant $\left(\chi^{2}(1)=3.07\right.$, $p=.080)$, and no main effects were significant $(p \mathrm{~s}>.14)$. Consistent with the findings in Experiments 1-5, the surprise stimuli induced a probe detection deficit, which gradually—but far from completely - habituated across trials. The SiD probe results for the control group were similar. 
A
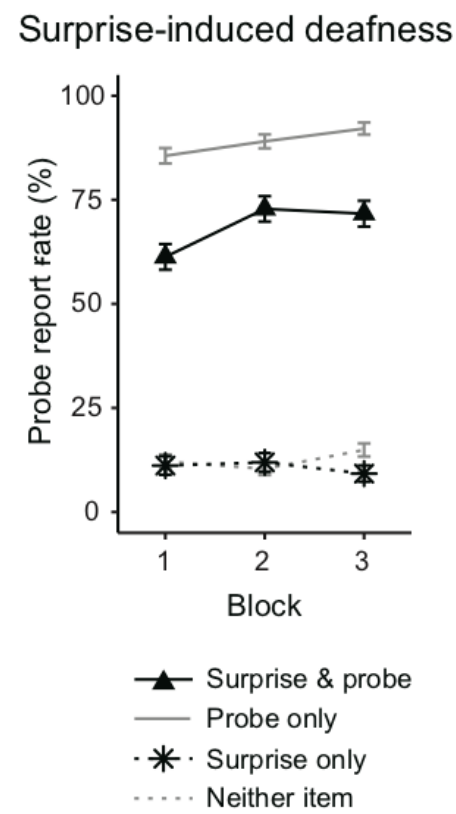

B

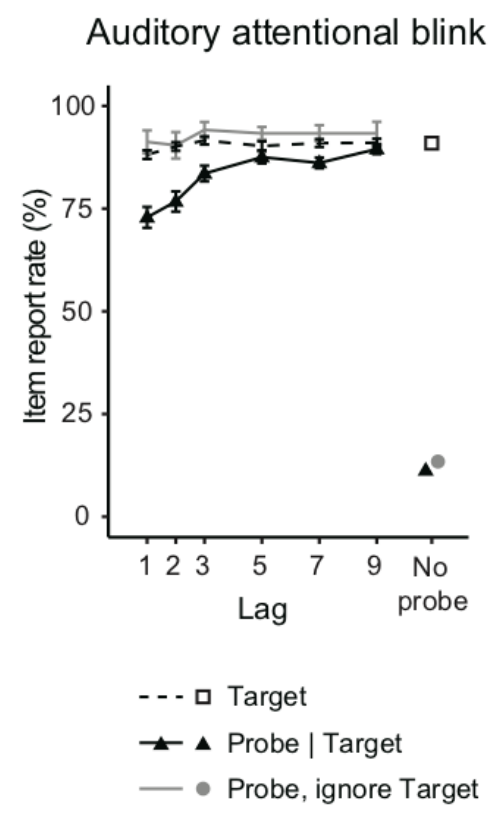

C

Correlation across deficits

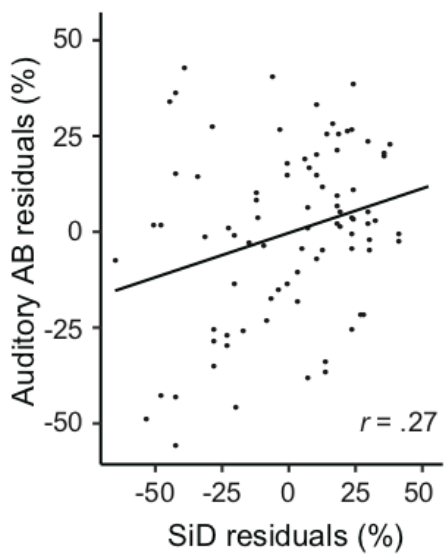

Figure 8. Performance in Experiment 7. A) Mean target detection rate for each trial type as a function of block for the experimental group. Note that all trials also contained a target, though performance was not calculated contingent on correct target discrimination. "Probe only" trials refer to Lag 9 trials, and "Neither item" trials refer to target only trials. B) Mean probe detection rate contingent on correct target discrimination as a function of lag. Data for the "Probe, ignore target" condition are from the control group, whereas other results are from the experimental group. C) Scatterplot of $\mathrm{SiD}$ and $\mathrm{AAB}$ residuals, showing the line of best fit. Error bars represent \pm 1 standard error of the mean (SEM). AAB $=$ auditory attentional blink. SiD = surprise-induced deafness.

\section{AAB: Target Discrimination}

Target discrimination was $90.06 \%(S D=9.42 \%)$ correct overall, showing that the task was manageable for most participants. Target discrimination also varied slightly but significantly by lag (Figure 8B; repeated-measures ANOVA: $F(5,645)=2.94, p=.01, \eta_{G}{ }^{2}$ $=.009$ ). Follow-up analysis revealed that the target discrimination rate at Lag 1 was 
significantly lower than at Lags 3, 7, and $9(p s<.036)$ but not significantly different from Lags 2 and $5(p s>$.19). Similar effects of Lag on target performance have been reported in other AAB studies (Martens et al., 2009; Shen \& Alain, 2012).

\section{AAB: Probe Reports}

Mean probe performance was calculated for each lag, conditional on correct target discrimination for the experimental group (probe | target; Figure 8B). Mean hit rates by participant were submitted to a mixed $6 \times 2$ ANOVA with lag as a within-subject variable and group (experimental, control) as a between-subject variable. This analysis revealed significant main effects of group $(F(1,148)=5.82, p=.017)$ and lag $(F(5,740)$ $=32.25, p<.001)$, as well as a significant interaction $(F(5,740)=11.70, p=.023)$. Performance improved with increasing lag for the experimental group (repeated-measures ANOVA: $\left.F(5,645)=32.94, p<.001, \eta_{G}^{2}=.071\right)$, but not for the control group $(F(5,95)$ $=0.51, p=.71)$. Compared to the control group, performance in the experimental group was significantly lower for Lags 1, 2, 3, and 7 (independent samples t-tests: $p \mathrm{~s}<.03$ ), though not for Lag $5(p=.06)$ or Lag $9(p=1)$. Finally, the false alarm rates between the two groups did not significantly differ $(t(148)=-0.42, p=.67)$. Overall, the results demonstrate a convincing $\mathrm{AAB}$, with a lag-dependent deficit that only appeared when the target was attended (Figure $8 \mathrm{~B})$.

To examine the possibility that target discrimination affected $\mathrm{SiD}$, we calculated the magnitude of the deficit for each participant in experimental and control groups. The probe hit rate during surprise trials was subtracted from this rate during standard Lag 9 trials. SiD magnitude for the experimental group (mean $=0.20, S D=0.25$ ) was not significantly different from that of control group (mean $=0.14, S D=0.23 ; t(148)=1.13, p=.26)$. The experimental group's SiD magnitude was also not significantly different from the Varying $20 \%$ condition of Experiment $3($ mean $=0.20, S D=0.19 ; t(198)=0.01, p=.99)$. We 
conclude that target discrimination did not interfere with perception of the surprise stimulus or its subsequent effects on probe detection.

\section{Preparation for Correlation Analyses}

A subset of the participant sample (46 of $130=36 \%$ ) had nearly perfect probe detection performance regardless of the condition. These individuals were removed for our primary correlation analyses, leaving 84 in the sample to be analyzed. As noted above, for completeness we also analyzed the full sample of 130 individuals and report those results.

\section{Internal-Consistency Reliability}

Metric stability was high for both $\mathrm{SiD}$ (split-half $r(82)=.74, p<.001$ ) and the AAB (split-half $r(82)=.65, \mathrm{p}<.001)$. These values represent high reliability and are comparable to those for the visual AB (Dale et al., 2013; Kelly \& Dux, 2011). Note that we did not correct our coefficients using the Spearman-Brown procedure (Spearman, 2010) because far more AAB-relevant trials contribute to the cross-deficit correlations that are of primary interest; therefore, the uncorrected $\mathrm{AAB}$ reliability values likely represent a sensible floor. When $\mathrm{SiD}$ residuals were instead calculated using Lag 7 and 9 trials as the baseline, similar reliability was obtained $(r(82)=.73, p<.001)$. When AAB residuals were calculated from Lag 3 trials, the reliability was considerably lower but still significant $(r(82)=.33, p=.010)$. The lower reliability is likely due to smaller overall $\mathrm{AAB}$ effects at Lag 3, though it is worth noting that the residuals from Lag 3 and the residuals from Lags 1 and 2 were highly correlated $(r(82)$ $=.62, p<.001)$. Finally, when the residuals were calculated from the complete sample (including individuals performing at ceiling), the reliability scores were again similar for both $\operatorname{SiD}(r(128)=.67, p<.001)$ and the AAB $(r(128)=.60, p<.001)$.

\section{Correlation between SiD and AAB Deficits}

To test the relationship between $\mathrm{SiD}$ and the $\mathrm{AAB}$, we calculated the correlation between the deficit magnitudes. A relatively small but significant correlation was found 
(Table 6). When SiD residuals were instead calculated using Lag 7 and 9 trials as the baseline, the SiD-AAB correlation was similar $(r(82)=.29, p=.008)$. The same was true when $\mathrm{AAB}$ residuals were calculated from Lag 3 trials $(r(82)=.28, p=.010)$, despite the much lower AAB reliability at Lag 3. Finally, when the residuals were calculated from the complete sample, the correlation was again comparable $(r(128)=.38, p<.001)$. Across different residual calculations, the correlation coefficient for $\mathrm{SiD}$ and the $\mathrm{AAB}$ indicated a small-to-moderate effect size.

Table 6. Correlation coefficients ( $r$-values) for different metrics considered pairwise in Experiment 7. The lack of correlation between probe detection and the deficit residuals is expected because baseline probe detection performance was statistically removed. ${ }^{*} p=.014$, $* * p<.001 . \mathrm{SiD}=$ surprise-induced deafness. $\mathrm{AAB}=$ auditory attentional blink.

\begin{tabular}{llll}
\hline Measure & 1 & 2 & 3 \\
\hline 1. Target discrimination & - & & \\
2. Probe detection (hit rate) & $.38^{* *}$ & - & \\
3. SiD magnitude & -.09 & .00 & - \\
4. AAB magnitude & -.16 & .05 & $.27^{*}$ \\
\hline
\end{tabular}

The magnitude of the SiD-AAB correlation was far lower than the reliability metrics for each deficit, suggesting a partial dissociation between the processing limitations behind $\mathrm{SiD}$ and the $\mathrm{AAB}$. This result is consistent with a partial dissociation between stimulus-driven and goal-directed attentional control that enjoys broad empirical support (Alho et al., 2015; Corbetta \& Shulman, 2002; Egeth \& Yantis, 1997). We further consider the meaning of both the partial overlap and partial dissociation in the General Discussion.

The present experiment contained a number of limitations that are also worth noting. 
The experiment was conducted in a single session online, precluding the collection of testretest measures across days. Such measures would be more compelling evidence of stable individual differences. Furthermore, the deficits were studied within the same paradigm, meaning that the SiD results were obtained from participants engaged in a dual-task situation (target discrimination followed by probe detection). We have addressed these limitations in a separate study in our laboratory, in which we found similar correlation magnitudes between SiD and the AAB, as well as their visual counterparts (Liaw et al., 2020).

\section{General Discussion}

Across seven experiments, we explored auditory attentional control using the surprise-induced deafness (SiD) paradigm. The first experiment established $\mathrm{SiD}$, in which a relatively rare and unexpected sound (the "surprise" stimulus) greatly impaired detection of a subsequent probe sound. Like the visual impairments in surprise-induced blindness (Asplund, Todd, Snyder, Gilbert, et al., 2010), SiD effects were time-dependent in two ways. First, probe detection was worst when the time between the surprise stimulus and the probe was relatively short, either $120 \mathrm{~ms}$ (Lag 1, no intervening distractors) or $360 \mathrm{~ms}$ (Lag 3, two intervening distractors). Second, the effect at Lag 3 habituated across tens of trials, whereas the Lag 1 effect persisted unchanged. Unlike its visual counterpart, SiD was largest at Lag 1 and was sensitive to stimulus type. SiD was observed when distractors and probes were pure tones but not when they were spoken letters.

Having established SiD, we then used the paradigm to test effect generalizability and ideas about informational surprise. Foremost, a simplified online experiment replicated the paradigm's key procedures and outcomes, allowing us to acquire large samples for subsequent experiments (Experiment 2). Both common sounds (including spoken letters) and non-auditory objects (e.g., complex tones) could induce SiD in an easier probe detection task (Experiment 4), further suggesting effect generalizability. SiD was also sensitive to surprise 
stimulus manipulations. Consistent with an Information Theory sense of "surprise", SiD was larger when surprise stimuli carried more information by varying across trials (Experiment $3)$.

We next used $\mathrm{SiD}$ to explore different forms of attentional control. SiD was observed even when probes were not feature singletons, indicating that the deficit was not due to participants adopting a goal-directed search strategy for singletons (Experiment 5). This result supports a stimulus-driven attentional capture account of SiD against a contingent capture one (Bacon \& Egeth, 1994; Egeth \& Yantis, 1997; Luck et al., 2020). We then developed and tested an auditory attentional blink (AAB) paradigm, in which the deficit was induced by goal-directed attentional search for a target (Experiment 6). This AAB paradigm was combined with the SiD paradigm in a hybrid experiment (Experiment 7). An individual differences approach showed that the two deficits were partially dissociable, indicating both shared and distinct attentional causes.

We conclude that the primary cause of surprise-induced deafness ( $\mathrm{SiD})$ is stimulusdriven attentional capture by potentially relevant, and therefore information-containing, stimuli. Nevertheless, additional attentional influences likely contribute to the deficit. Below we explore the theoretical context and implications of our work, SiD's relationship to other attentional capture paradigms, and potential avenues for future exploration.

\section{Goal-directed and stimulus-driven attention}

Traditionally, attentional control has been conceptualized in two forms (Corbetta \& Shulman, 2002; Egeth \& Yantis, 1997; Fritz et al., 2007). Goal-directed control reflects context knowledge or voluntary deployment, whereas stimulus-driven control comes from events and stimuli in the environment. Surprise-induced deafness (SiD) most clearly tests and demonstrates stimulus-driven attentional control, as an unexpected item rapidly and automatically reallocates attention. In electrophysiological studies, such task-irrelevant 
sounds elicit a novelty-P3 response, which differs from the goal-related target-P3 in its habituation, more rapid onset, and more frontal scalp distribution (Debener et al., 2002). Despite such behavioral and neural distinctions, however, the SiD paradigm also reflects goal-directed attentional control; the $\mathrm{SiD}$ deficit is the consequence of its disruption.

Coherent behavior requires interaction between the two types of attentional control (Asplund, Todd, Snyder, Gilbert, et al., 2010; Egeth \& Yantis, 1997; Serences et al., 2005). This interaction may be achieved via overlapping neurocognitive processes. Although some studies suggest the relative independence of goal-directed and stimulus-driven attentional control (Pinto et al., 2013), others argue that they cannot function in isolation (Rauschenberger, 2010) or that they involve the same neural circuitry (Buschman \& Miller, 2007). The consensus view stakes out a middle ground: The two forms of attentional control are partially dissociable, neurally and psychologically (Asplund, Todd, Snyder, \& Marois, 2010; Corbetta \& Shulman, 2002; Serences et al., 2005). Neuroimaging studies of auditory attention have identified distinct neural correlates for each form of attentional control, as well as overlaps in the temporo-parietal, superior parietal, and frontal cortex (Alho et al., 2015; Salmi et al., 2009). Shared neurocognitive processes may explain how unexpected stimuli disrupt ongoing tasks in the SiD and similar paradigms. They may also account for the relationship between the $\mathrm{AAB}$ and $\mathrm{SiD}$ deficits in Experiment 7, as individual differences in processes that contribute to both forms of attentional control would lead to correlated deficits. Importantly, the observed relationship was not simply due to probe detection ability, as individual differences in this goal-directed component were removed through regression.

Another possibility is that the $\mathrm{SiD}$ deficit reflects a sequence of stimulus-driven and goal-directed attentional processes. Horváth et al. (2008) describe auditory distraction using a serial model that includes 1) detection of an unexpected stimulus, 2) orienting attention towards the stimulus, and 3) recovery from distraction. SiD likely involves this same 
sequence of processes; indeed, the orienting response (OR) has been identified with the second process, and both evoke a P3a electrophysiological response (Horváth et al., 2008; Parmentier, 2014; Sokolov, 2002). The third process is thought to involve attentional reorienting from a task-irrelevant sound back to task-relevant stimuli (Sussman et al., 2003). This recovery from distraction is indexed by the reorienting negativity (RON), an electrophysiological deflection that peaks approximately $500 \mathrm{~ms}$ after the distracting sound's onset. Given its timecourse, the RON may represent attentional processes that could curtail SiD's duration and severity. The RON also indexes cue-dependent task set switches, consistent with its representing goal-directed attentional control (Hölig \& Berti, 2010). Taskswitch cues also evoke early sensory components and a P3a, however, so these electrophysiological markers should not be interpreted as having a one-to-one mapping with attentional processes (Horváth et al., 2008; Parmentier, 2014).

Attentional reorienting in auditory distraction is a reactive process, invoked in response to an unexpected stimulus. Other reactive processes may also be involved in $\mathrm{SiD}$, with some contributing more directly to the observed deficit. For example, Theeuwes et al. (2000) suggest that stimuli can capture attention in a stimulus-driven fashion, after which they may prolong the disruption if subsequent processes engage with them —or fail to disengage attention from them. As the initial stimulus-driven attentional capture may last only $\sim 150 \mathrm{~ms}$, the $\mathrm{SiD}$ effect reported here may critically rely on these subsequent processes, which may include goal-directed investigation of potentially relevant stimuli and events (Luck et al., 2020; Theeuwes et al., 2000). Conversely, the detection deficits we observed when probe items closely followed surprise stimuli $(120 \mathrm{~ms})$ may reflect stimulus-driven factors alone, similar to what was observed and argued for surprise-induced blindness (Asplund, Todd, Snyder, Gilbert, et al., 2010).

In addition to reactive processes, proactive attentional settings may modulate $\mathrm{SiD}$. 
Knowledge of potential distraction may lead to modified goal-directed attentional settings, including proactive suppression of certain stimuli. Rather little is known, however, about proactive suppression in auditory distraction tasks. Direct instruction at the beginning of a task block can reduce the effects of salient stimuli in an AAB context (Horváth \& Burgyán, 2011), but most proactive suppression effects are more circumscribed. Predictive visual cues, which precede the distracting stimuli by a few hundred milliseconds, can reduce auditory distraction in general or from specific deviants (Horváth et al., 2011; Sussman et al., 2003). Accompanying electrophysiological markers such as the P3a and RON, however, persist even as behavioral distraction is attenuated (Sussman et al., 2003). Reduced distraction from anticipation or cues is also inconsistently evidenced across paradigms (Bidet-Caulet et al., 2015).

Although the precise circumstances under which distraction can be prevented or successfully overcome remain unclear, participants completing an SiD task manage to do so. Moreover, their attentional settings change as they learn the identities of surprise stimuli and their frequency of occurrence. Such learning leads to rapid habituation of the deficit. But is the attentional influence of this learning goal-directed or stimulus-driven?

\section{Beyond the attentional control dichotomy}

Awh et al. (2012) argue that conceptualizing attentional control as either goaldirected or stimulus-driven is incomplete and often unhelpful. They point to effects in which visuospatial attention is directed neither by physical stimulus features nor task goals. For example, in a contingent capture experiment in which the target-relevant color changed on each trial, attention was still captured by irrelevant distractors with a recently-relevant color (Belopolsky et al., 2010). Furthermore, when individuals learned that a color indicated reward availability, that color continued to capture attention even after that reward contingency no longer existed (Pearson \& Le Pelley, 2020). Such examples indicate that 
attention is influenced by selection history. This influence often works against behavioral goals and voluntary attentional control.

Although selection history may be unconscious and automatic, it nevertheless represents attentional control arising from knowledge and expectations. Such knowledge and expectations are learned in the context of the task. For example, participants can learn to suppress the distracting effects of a specific color singleton (Gaspelin \& Luck, 2019). Explicit instruction about the distracting color does not appear to be effective; instead, suppressive effects build gradually through experience with the singleton color. Similarly, reward values that influence attention appear to be learned through implicit associations, both instrumental and Pavlovian (Pearson \& Le Pelley, 2020). Although there is debate about whether attentional influences selection history should be considered goal-directed, they are importantly different from conscious, voluntary attentional control (Luck et al., 2020).

The experiments referenced above involve visuospatial attention and color distractors, but the resulting theories may also apply to auditory attentional control as explored through the surprise-induced deafness paradigm. In SiD, relatively rare stimuli capture attention because of their informational surprise, which represents a violation of ongoing expectations about the world (Asplund et al., 2020; Asplund, Todd, Snyder, Gilbert, et al., 2010; Horstmann, 2015; Meyer et al., 1991; Niepel et al., 1994). The stimuli also induce Bayesian surprise because participants change their behavior (reduced distraction) and beliefs as they learn about the distracting stimuli (Itti \& Baldi, 2009). Both explicit and implicit learning likely occur, though which knowledge ultimately influences behavioral performance is a question left for future research. A clue, however, can be found in SiD's visual counterpart: Surprise-induced blindness is little affected by direct instruction that rare stimuli will appear during the task (Asplund, Todd, Snyder, Gilbert, et al., 2010).

It is therefore possible that the $\mathrm{SiD}$ deficit and its habituation reflect selection 
history's influence on temporal auditory attention. When stimuli are automatically assessed to be relatively novel and unexpected, attention's engagement could be reactively prolonged (Theeuwes et al., 2000). Alternatively, reactive attentional suppression or disengagement may build across surprise trials, thereby reducing the capture effects (Gaspelin \& Luck, 2019; Luck et al., 2020). Distractor suppression or adjusted attentional focus could also be proactive. Reaction times increase for trials following visual (Asplund, Todd, Snyder, \& Marois, 2010) or auditory (Parmentier et al., 2011) distraction, perhaps reflecting implicit attentional adjustments. On the whole, however, we find the first possibility most likely. Inhibitory markers have not been observed in temporal attention experiments (Asplund, Todd, Snyder, \& Marois, 2010; Horváth et al., 2008; Sussman et al., 2003), and adjustments that affect probe detection performance tend to last for only a handful of subsequent trials (Asplund, Todd, Snyder, \& Marois, 2010; Parmentier et al., 2011).

Comparisons of surprise deficits and their habituation rates across modalities could reveal more about attentional control and relevant learning mechanisms. For example, SiD and surprise-induced blindness have broadly similar deficit timecourses within and across surprise trials (Asplund, Todd, Snyder, Gilbert, et al., 2010). Such similarities may reflect supramodal alerting, which cross-modal surprise experiments could investigate (Asplund et al., 2020; Horstmann, 2015; Parmentier, 2014). Alternatively, the deficits may reflect modality-specific attentional control processes that function analogously across vision and audition. The slower habituation rates in audition, however, could indicate modality differences in learning or the attentional consequences of learning; low-level procedural differences between the paradigms provide an alternative explanation. Surprise deficit paradigms that are designed to be as procedurally similar as possible could help to distinguish between these accounts. A complement is an individual differences approach, which we explore elsewhere (Liaw et al., 2020). 
Comparative approaches can also be used to understand the affective aspects of surprise and their consequences for attentional control. Unexpected events cause emotional responses in addition to cognitive ones, a fact reflected in both the common use of the word "surprise" and the empirical literature (Meyer et al., 1991; Niepel et al., 1994). One potential comparison paradigm is emotion-induced blindness (or an auditory analogue), in which an affectively arousing stimulus disrupts perception (McHugo et al., 2013). The attentional responses to affective stimuli (including aversive ones such as unpleasant images or thermal pain) may be similar to responses to neutral stimuli. Conversely, they may involve additional biasing processes or learning mechanisms.

In summary, surprise-induced deafness is a novel perceptual phenomenon caused by stimulus-driven attentional capture. The deficit's sensitivity to information content and its habituation illustrate other attentional influences on behavior and awareness. Such influences include goal-directed and stimulus-driven attentional control processes, as well as implicitlylearned internal models that may go beyond the traditional dichotomy of attentional control.

\section{References}

Alho, K., Salmi, J., Koistinen, S., Salonen, O., \& Rinne, T. (2015). Top-down controlled and bottom-up triggered orienting of auditory attention to pitch activate overlapping brain networks. Brain Research, 1626, 136-145. https://doi.org/10.1016/j.brainres.2014.12.050

Arnell, K. M., \& Jenkins, R. (2004). Revisiting within-modality and cross-modality attentional blinks: Effects of target—distractor similarity. Perception \& Psychophysics, 66(7), 
Arnell, K. M., \& Jolicoeur, P. (1999). The attentional blink across stimulus modalities: Evidence for central processing limitations. Journal of Experimental Psychology. Human Perception and Performance, 25(3), 630-648.

Asplund, C. L., Obana, T., Bhatnagar, P., Koh, X. Q., \& Perrault, S. T. (2020). It's All in the Timing: Principles of Transient Distraction Illustrated with Vibrotactile Tasks. $A C M$ Transactions on Computer-Human Interaction, 27(3), 1-29. https://doi.org/10.1145/3386358

Asplund, C. L., Todd, J. J., Snyder, A. P., Gilbert, C. M., \& Marois, R. (2010). Surprise-induced blindness: A stimulus-driven attentional limit to conscious perception. Journal of Experimental Psychology: Human Perception and Performance, 36(6), 1372-1381. https://doi.org/10.1037/a0020551

Asplund, C. L., Todd, J. J., Snyder, A. P., \& Marois, R. (2010). A central role for the lateral prefrontal cortex in goal-directed and stimulus-driven attention. Nature Neuroscience, 13(4), 507-512. https://doi.org/10.1038/nn.2509

Awh, E., Belopolsky, A. V., \& Theeuwes, J. (2012). Top-down versus bottom-up attentional control: A failed theoretical dichotomy. Trends in Cognitive Sciences, 16(8), 437-443. https://doi.org/10.1016/j.tics.2012.06.010

Bacon, W. F., \& Egeth, H. E. (1994). Overriding stimulus-driven attentional capture. Perception \& Psychophysics, 55(5), 485-496. https://doi.org/10.3758/BF03205306 
Barr, D. J., Levy, R., Scheepers, C., \& Tily, H. J. (2013). Random effects structure for confirmatory hypothesis testing: Keep it maximal. Journal of Memory and Language, 68(3), 255-278. https://doi.org/10.1016/j.jml.2012.11.001

Bates, D., Mächler, M., Bolker, B., \& Walker, S. (2015). Fitting Linear Mixed-Effects Models Using Ime4. Journal of Statistical Software, 67(1). https://doi.org/10.18637/jss.v067.i01

Belopolsky, A. V., Schreij, D., \& Theeuwes, J. (2010). What is top-down about contingent capture? Attention, Perception, \& Psychophysics, 72(2), 326-341. https://doi.org/10.3758/APP.72.2.326

Bidet-Caulet, A., Bottemanne, L., Fonteneau, C., Giard, M.-H., \& Bertrand, O. (2015). Brain Dynamics of Distractibility: Interaction Between Top-Down and Bottom-Up Mechanisms of Auditory Attention. Brain Topography, 28(3), 423-436. https://doi.org/10.1007/s10548-014-0354-x

Bucker, B., \& Theeuwes, J. (2017). Pavlovian reward learning underlies value driven attentional capture. Attention, Perception, \& Psychophysics, 79(2), 415-428. https://doi.org/10.3758/s13414-016-1241-1

Buschman, T. J., \& Miller, E. K. (2007). Top-down versus Bottom-up Control of Attention in the Prefrontal and Posterior Parietal Cortices. Science, New Series, 315(5820), 18601862. 
Chun, M. M., \& Marois, R. (2002). The dark side of visual attention. Current Opinion in Neurobiology, 12(2), 184-189. https://doi.org/10.1016/S0959-4388(02)00309-4

Corbetta, M., \& Shulman, G. L. (2002). Control of goal-directed and stimulus-driven attention in the brain. Nature Reviews Neuroscience, 3(3), 201-215. https://doi.org/10.1038/nrn755

Courchesne, E., Hillyard, S. A., \& Galambos, R. (1975). Stimulus novelty, task relevance and the visual evoked potential in man. Electroencephalography and Clinical Neurophysiology, 39(2), 131-143. https://doi.org/10.1016/0013-4694(75)90003-6

Crowder, R. G. (1993). Auditory memory. In S. McAdams \& E. Bigand (Eds.), Thinking in sound: The cognitive psychology of human audition (pp. 113-145). Oxford University Press.

Crump, M. J. C., McDonnell, J. V., \& Gureckis, T. M. (2013). Evaluating Amazon's Mechanical Turk as a Tool for Experimental Behavioral Research. PLoS ONE, 8(3), e57410. https://doi.org/10.1371/journal.pone.0057410

Dale, G., Dux, P. E., \& Arnell, K. M. (2013). Individual differences within and across attentional blink tasks revisited. Attention, Perception, \& Psychophysics, 75(3), 456467. https://doi.org/10.3758/s13414-012-0415-8

Dalton, P., \& Lavie, N. (2004). Auditory Attentional Capture: Effects of Singleton Distractor Sounds. Journal of Experimental Psychology: Human Perception and Performance, 
30(1), 180-193. https://doi.org/10.1037/0096-1523.30.1.180

Debener, S., Kranczioch, C., Herrmann, C. S., \& Engel, A. K. (2002). Auditory novelty oddball allows reliable distinction of top-down and bottom-up processes of attention. International Journal of Psychophysiology, 46(1), 77-84. https://doi.org/10.1016/S0167-8760(02)00072-7

Delahaye, R., Fantini, D. A., \& Meddis, R. (1999). Effects of practice on performance for different masking tasks. The Journal of the Acoustical Society of America, 105(2), 1153-1153. https://doi.org/10.1121/1.425482

Donchin, E. (1981). Surprise!...Surprise? Psychophysiology, 18(5), 493-513.

Downar, J., Crawley, A. P., Mikulis, D. J., \& Davis, K. D. (2002). A Cortical Network Sensitive to Stimulus Salience in a Neutral Behavioral Context Across Multiple Sensory Modalities. Journal of Neurophysiology, 87(1), 615-620. https://doi.org/10.1152/jn.00636.2001

Dux, P. E., \& Marois, R. (2009). The attentional blink: A review of data and theory. Attention, Perception \& Psychophysics, 71(8), 1683-1700. https://doi.org/10.3758/APP.71.8.1683

Egeth, H. E., \& Yantis, S. (1997). Visual attention: Control, representation, and time course. Annual Review of Psychology, 48(1), 269-297.

Escera, C., Alho, K., Schröger, E., \& Winkler, I. W. (2000). Involuntary Attention and 
Distractibility as Evaluated with Event-Related Brain Potentials. Audiology and Neurotology, 5(3-4), 151-166. https://doi.org/10.1159/000013877

Escera, C., Alho, K., Winkler, I., \& Näätänen, R. (1998). Neural Mechanisms of Involuntary Attention to Acoustic Novelty and Change. Journal of Cognitive Neuroscience, 10(5), 590-604. https://doi.org/10.1162/089892998562997

Folk, C. L., Remington, R. W., \& Johnston, J. C. (1992). Involuntary covert orienting is contingent on attentional control settings. Journal of Experimental Psychology. Human Perception and Performance, 18(4), 1030-1044.

Font, F., Roma, G., \& Serra, X. (2013). Freesound technical demo. Proceedings of the 21st ACM International Conference on Multimedia - MM '13, 411-412. https://doi.org/10.1145/2502081.2502245

Fox, J., \& Weisberg, S. (2011). An $R$ Companion to Applied Regression. SAGE. http://socserv.socsci.mcmaster.ca/jfox/Books/Companion

Friedman, D., Cycowicz, Y. M., \& Gaeta, H. (2001). The novelty P3: An event-related brain potential (ERP) sign of the brain's evaluation of novelty. Neuroscience \& Biobehavioral Reviews, 25(4), 355-373. https://doi.org/10.1016/S0149-7634(01)00019-7

Friedman, D., \& Simpson, G. V. (1994). ERP amplitude and scalp distribution to target and novel events: Effects of temporal order in young, middle-aged and older adults. Cognitive Brain Research, 2(1), 49-63. https://doi.org/10.1016/0926-6410(94)90020-5 
Fritz, J. B., Elhilali, M., David, S. V., \& Shamma, S. A. (2007). Auditory attention-Focusing the searchlight on sound. Current Opinion in Neurobiology, 17(4), 437-455. https://doi.org/10.1016/j.conb.2007.07.011

Gaspelin, N., \& Luck, S. J. (2019). Inhibition as a potential resolution to the attentional capture debate. Current Opinion in Psychology, 29, 12-18. https://doi.org/10.1016/j.copsyc.2018.10.013

Hölig, C., \& Berti, S. (2010). To switch or not to switch: Brain potential indices of attentional control after task-relevant and task-irrelevant changes of stimulus features. Brain Research, 1345, 164-175. https://doi.org/10.1016/j.brainres.2010.05.047

Horstmann, G. (2005). Attentional Capture by an Unannounced Color Singleton Depends on Expectation Discrepancy. Journal of Experimental Psychology: Human Perception and Performance, 31(5), 1039-1060. https://doi.org/10.1037/0096-1523.31.5.1039

Horstmann, G. (2015). The surprise-attention link: A review: The surprise-attention link. Annals of the New York Academy of Sciences, 1339(1), 106-115. https://doi.org/10.1111/nyas.12679

Horváth, J., \& Burgyán, A. (2011). Distraction and the auditory attentional blink. Attention, Perception, \& Psychophysics, 73(3), 695-701. https://doi.org/10.3758/s13414-0100077-3

Horváth, J., Sussman, E., Winkler, I., \& Schröger, E. (2011). Preventing distraction: Assessing 
stimulus-specific and general effects of the predictive cueing of deviant auditory events. Biological Psychology, 87(1), 35-48. https://doi.org/10.1016/j.biopsycho.2011.01.011 Horváth, J., \& Winkler, I. (2010). Distraction in a continuous-stimulation detection task. Biological Psychology, $\quad$ 83(3), 229-238. https://doi.org/10.1016/j.biopsycho.2010.01.004

Horváth, J., Winkler, I., \& Bendixen, A. (2008). Do N1/MMN, P3a, and RON form a strongly coupled chain reflecting the three stages of auditory distraction? Biological Psychology, 79(2), 139-147. https://doi.org/10.1016/j.biopsycho.2008.04.001

Itti, L., \& Baldi, P. (2009). Bayesian surprise attracts human attention. Vision Research, 49(10), 1295-1306. https://doi.org/10.1016/j.visres.2008.09.007

Jesteadt, W., Bacon, S. P., \& Lehman, J. R. (1982). Forward masking as a function of frequency, masker level, and signal delay. The Journal of the Acoustical Society of America, 71(4), 950-962. https://doi.org/10.1121/1.387576

Kahneman, D. (1973). Attention and effort. Prentice-Hall.

Kelly, A. J., \& Dux, P. E. (2011). Different attentional blink tasks reflect distinct information processing limitations: An individual differences approach. Journal of Experimental Psychology: Human Perception and Performance, 37(6), 1867-1873. https://doi.org/10.1037/a0025975

Knight, R. T., \& Scabini, D. (1998). Anatomic Bases of Event-Related Potentials and Their 
Relationship to Novelty Detection in Humans: Journal of Clinical Neurophysiology, 15(1), 3-13. https://doi.org/10.1097/00004691-199801000-00003

Lawrence, M., A. (2016). ez: Easy Analysis and Visualization of Factorial Experiments. https://CRAN.R-project.org/package=ez

Lenth, R., Singmann, H., Love, J., Buerkner, P., \& Herve, M. (2019). emmeans: Estimated Marginal Means, aka Least-Square Means (1.3.1) [R; $\mathrm{R} \quad(>=3.2)]$. https://github.com/rvlenth/emmeans

Liaw, G. J., Chia, T. T. Y., Obana, T., \& Asplund, C. L. (2020). Do temporal attentional limitations share cognitive causes? A correlational study of different attentional forms and modalities.

Luck, S. J., Gaspelin, N., Folk, C. L., Remington, R. W., \& Theeuwes, J. (2020). Progress toward resolving the attentional capture debate. Visual Cognition, 0(0), 1-21. https://doi.org/10.1080/13506285.2020.1848949

MacLean, M. H., \& Arnell, K. M. (2012). A conceptual and methodological framework for measuring and modulating the attentional blink. Attention, Perception, \& Psychophysics, 74(6), 1080-1097. https://doi.org/10.3758/s13414-012-0338-4

Maljkovic, V., \& Nakayama, K. (1994). Priming of pop-out: I. Role of features. Memory \& Cognition, 22(6), 657-672. https://doi.org/10.3758/BF03209251

Martens, S., Johnson, A., Bolle, M., \& Borst, J. (2009). A Quick Visual Mind Can be a Slow 
Auditory Mind: Individual Differences in Attentional Selection Across Modalities. Experimental Psychology, 56(1), 33-40. https://doi.org/10.1027/1618-3169.56.1.33

Martens, S., Kandula, M., \& Duncan, J. (2010). Restricted Attentional Capacity within but Not between Sensory Modalities: An Individual Differences Approach. PLoS ONE, 5(12), e15280. https://doi.org/10.1371/journal.pone.0015280

McHugo, M., Olatunji, B. O., \& Zald, D. H. (2013). The emotional attentional blink: What we know so far. Frontiers in Human Neuroscience, 7. https://doi.org/10.3389/fnhum.2013.00151

Meyer, W.-U., Niepel, M., Rudolph, U., \& Schützwohl, A. (1991). An experimental analysis of surprise. Cognition $\quad \& \quad$ Emotion, $295-311$. https://doi.org/10.1080/02699939108411042

Mondor, T. A. (1998). A transient processing deficit following selection of an auditory target. Psychonomic Bulletin \& Review, 5(2), 305-311. https://doi.org/10.3758/BF03212956

Moore, B. C. J., \& Glasberg, B. R. (1981). Auditory filter shapes derived in simultaneous and forward masking. The Journal of the Acoustical Society of America, 70(4), 1003-1014. https://doi.org/10.1121/1.386950

Näätänen, R., \& Alho, K. (1995). Mismatch negativity-a unique measure of sensory processing in audition. International Journal of Neuroscience, 80(1-4), 317-337. https://doi.org/10.3109/00207459508986107 
Näätänen, R., Paavilainen, P., Rinne, T., \& Alho, K. (2007). The mismatch negativity (MMN) in basic research of central auditory processing: A review. Clinical Neurophysiology, 118(12), 2544-2590. https://doi.org/10.1016/j.clinph.2007.04.026

Niepel, M., Rudolph, U., Schützwohl, A., \& Meyer, W.-U. (1994). Temporal characteristics of the surprise reaction induced by schema-discrepant visual and auditory events. Cognition \& Emotion, 8(5), 433-452. https://doi.org/10.1080/02699939408408951

Parmentier, F. B. R. (2014). The cognitive determinants of behavioral distraction by deviant auditory stimuli: A review. Psychological Research, 78(3), 321-338. https://doi.org/10.1007/s00426-013-0534-4

Parmentier, F. B. R., Elsley, J. V., Andrés, P., \& Barceló, F. (2011). Why are auditory novels distracting? Contrasting the roles of novelty, violation of expectation and stimulus change. Cognition, 119(3), 374-380. https://doi.org/10.1016/j.cognition.2011.02.001

Pavlov, I. P. (1927). Conditioned reflexes. Oxford University Press.

Pearson, D., \& Le Pelley, M. E. (2020). Learning to avoid looking: Competing influences of reward on overt attentional selection. Psychonomic Bulletin \& Review, 27(5), 998-1005. https://doi.org/10.3758/s13423-020-01770-3

Peer, E., Vosgerau, J., \& Acquisti, A. (2014). Reputation as a sufficient condition for data quality on Amazon Mechanical Turk. Behavior Research Methods, 46(4), 1023-1031. https://doi.org/10.3758/s13428-013-0434-y 
Peirce, J. W. (2007). PsychoPy_Psychophysics software in Python. Journal of Neuroscience Methods, 162(1), 8-13. https://doi.org/10.1016/j.jneumeth.2006.11.017

Pinto, Y., van der Leij, A. R., Sligte, I. G., Lamme, V. A. F., \& Scholte, H. S. (2013). Bottomup and top-down attention are independent. Journal of Vision, 13(3), 16-16. https://doi.org/10.1167/13.3.16

Potter, M. C., Chun, M. M., Banks, B. S., \& Muckenhoupt, M. (1998). Two Attentional Deficits in Serial Target Search: The Visual Attentional Blink and an Amodal Task-Switch Deficit. Journal of Experimental Psychology: Learning, Memory, and Cognition, 24(4), 979-992.

Rauschenberger, R. (2010). Reentrant processing in attentional guidance-Time to abandon old dichotomies. Acta Psychologica, 135(2), 109-111. https://doi.org/10.1016/j.actpsy.2010.04.014

Roeber, U., Berti, S., \& Schröger, E. (2003). Auditory distraction with different presentation rates: An event-related potential and behavioral study. Clinical Neurophysiology, 114(2), 341-349. https://doi.org/10.1016/S1388-2457(02)00377-2

Ruthruff, E., \& Pashler, H. (2001). Perceptual and central interference in dual-task performance. In K. Shapiro (Ed.), The limits of attention: Temporal constraints in human information processing (pp. 100-123). Oxford University Press.

Salmi, J., Rinne, T., Koistinen, S., Salonen, O., \& Alho, K. (2009). Brain networks of bottom- 
up triggered and top-down controlled shifting of auditory attention. Brain Research, 1286, 155-164. https://doi.org/10.1016/j.brainres.2009.06.083

Scharf, B. (1998). Auditory attention: The psychoacoustical approach. In H. Pashler (Ed.), Attention (pp. 75-117). Psychology Press.

Schröger, E. (1996). A Neural Mechanism for Involuntary Attention Shifts to Changes in Auditory Stimulation. Journal of Cognitive Neuroscience, 8(6), 527-539. https://doi.org/10.1162/jocn.1996.8.6.527

Serences, J. T., Shomstein, S., Leber, A. B., Golay, X., Egeth, H. E., \& Yantis, S. (2005). Coordination of voluntary and stimulus-driven attentional control in human cortex. Psychological Science, 16(2), 114-122.

Shen, D., \& Alain, C. (2012). Implicit Temporal Expectation Attenuates Auditory Attentional Blink. PLoS ONE, 7(4), e36031. https://doi.org/10.1371/journal.pone.0036031

Shen, D., \& Mondor, T. A. (2006). Effect of distractor sounds on the auditory attentional blink. Perception \& Psychophysics, 68(2), 228-243. https://doi.org/10.3758/BF03193672

Shen, D., \& Mondor, T. A. (2008). Object file continuity and the auditory attentional blink. Perception \& Psychophysics, 70(5), 896-915. https://doi.org/10.3758/PP.70.5.896

Shore, D. I., McLaughlin, E. N., \& Klein, R. M. (2001). Modulation of the attentional blink by differential resource allocation. Canadian Journal of Experimental Psychology/Revue Canadienne de Psychologie Expérimentale, 55(4), 318-324. 
https://doi.org/10.1037/h0087379

Sokolov, E. N. (1990). The orienting response, and future directions of its development. The Pavlovian Journal of Biological Science, 25(3), 142-150. https://doi.org/10.1007/BF02974268

Sokolov, E. N. (Ed.). (2002). The orienting response in information processing. L. Erlbaum Associates.

Spearman, C. (2010). The proof and measurement of association between two things. International Journal of Epidemiology, 39(5), 1137-1150. https://doi.org/10.1093/ije/dyq191

Stanislaw, H., \& Todorov, N. (1999). Calculation of signal detection theory measures. Behavior Research Methods, Instruments, \& Computers, 31(1), 137-149. https://doi.org/10.3758/BF03207704

Summerville, A., \& Chartier, C. R. (2013). Pseudo-dyadic “interaction" on Amazon's Mechanical Turk. Behavior Research Methods, 45(1), 116-124. https://doi.org/10.3758/s13428-012-0250-9

Sussman, E., Winkler, I., \& Schröger, E. (2003). Top-down control over involuntary attention switching in the auditory modality. Psychonomic Bulletin \& Review, 10(3), 630-637. https://doi.org/10.3758/BF03196525

Theeuwes, J., Atchley, P., \& Kramer, A. F. (2000). On the time course of top-down and bottom- 
up control of visual attention. In S. Monsell \& J. Driver (Eds.), Control of Cognitive Processes: Attention and Performance Xviii (ISI:000175879400004; pp. 105-124). MIT Press. ://000175879400004

Tremblay, S., Vachon, F., \& Jones, D. M. (2005). Attentional and perceptual sources of the auditory attentional blink. Perception \& Psychophysics, 67(2), 195-208.

Vachon, F., Hughes, R. W., \& Jones, D. M. (2012). Broken expectations: Violation of expectancies, not novelty, captures auditory attention. Journal of Experimental Psychology: Learning, Memory, and Cognition, 38(1), 164-177. https://doi.org/10.1037/a0025054

Vachon, F., Labonté, K., \& Marsh, J. E. (2017). Attentional capture by deviant sounds: A noncontingent form of auditory distraction? Journal of Experimental Psychology: Learning, Memory, and Cognition, 43(4), 622-634. https://doi.org/10.1037/xlm0000330

Vachon, F., \& Tremblay, S. (2005). Auditory Attentional Blink: Masking the Second Target is Necessary, Delayed Masking is Sufficient. Canadian Journal of Experimental Psychology/Revue Canadienne de Psychologie Expérimentale, 59(4), 279-286. https://doi.org/10.1037/h0087480

Vachon, F., \& Tremblay, S. (2006). Delayed Masking and the Auditory Attentional Blink: A Test for Retrieval Competition and Bottleneck Models. Experimental Psychology, 
Surprise-induced Deafness

53(3), 182-190. https://doi.org/10.1027/1618-3169.53.3.182

Vachon, F., Tremblay, S., Hughes, R. W., \& Jones, D. M. (2009). Capturing and Unmasking the Mask in the Auditory Attentional Blink. Experimental Psychology, 57(5), 346-353. https://doi.org/10.1027/1618-3169/a000041 